\title{
An Algorithm for the Extraction of Tafel Slopes
}

\author{
Peter Agbo ${ }^{\dagger * *}$ and Nemanja Danilovic ${ }^{\#}$
}

Chemical Sciences Division ${ }^{\dagger}$, Joint Center for Artificial Photosynthesis ${ }^{\ddagger}$, Energy Systems and Distributed Resources Division", Lawrence Berkeley National Laboratory, Berkeley CA 94720

*Corresponding author

\section{Supplementary Information}

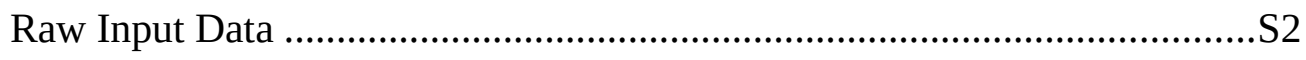

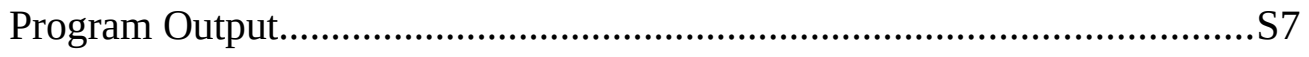

Influence of $\mathrm{R}^{2}$ Thresholds (OER examples) .........................................S9

Influence of $\mathrm{d \eta}$ Thresholds (OER examples)........................................S10

Influence of Binning (OER examples).................................................S11

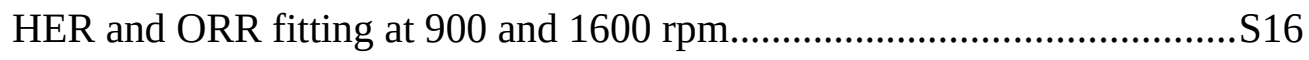

Optimization of the Low-overpotential Tafel Region in ORR..................S18

Table of OER, HER and ORR Binning Results.....................................S19 


\section{Raw Input Data}

Iridium-catalyzed Oxygen Evolution Reaction (OER)

$0.1 \mathrm{M} \mathrm{HClO}_{4}$
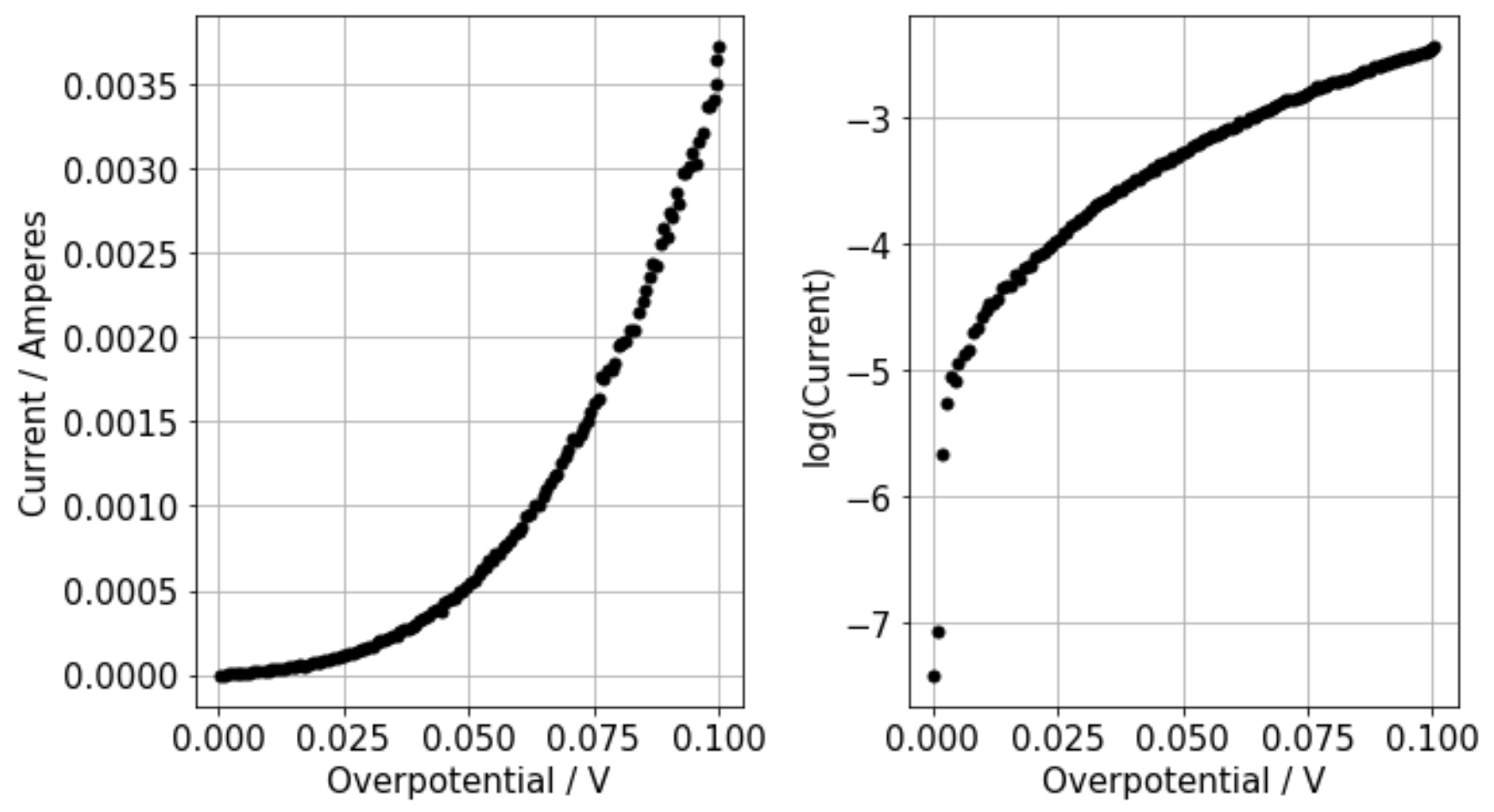

Figure S1

1600 rpm rotation 
Platinum-catalyzed Hydrogen Evolution Reaction (HER)

$0.1 \mathrm{M} \mathrm{HClO}_{4}$

Multiple rotation rates:
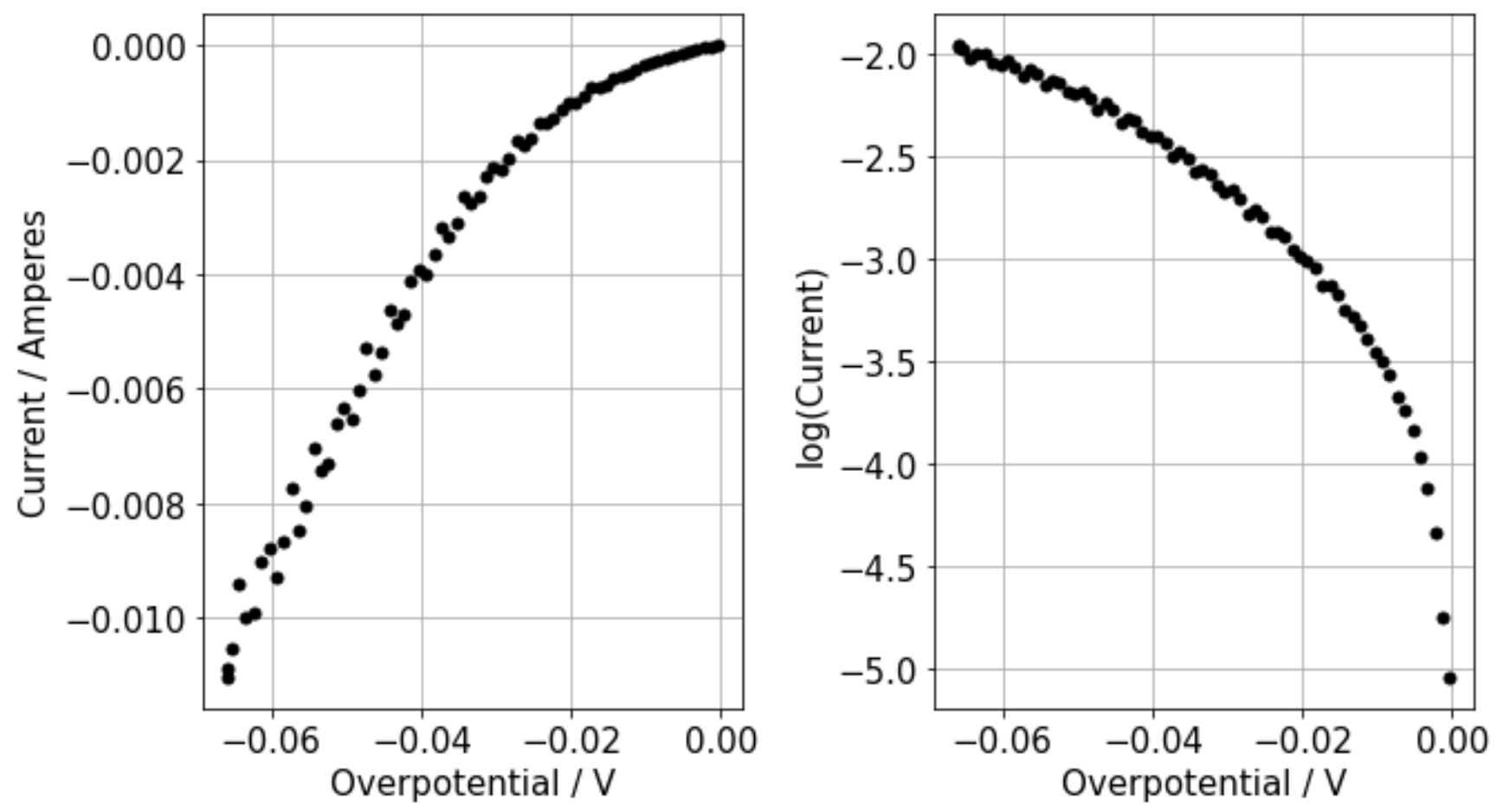

Figure S2, 900 rpm
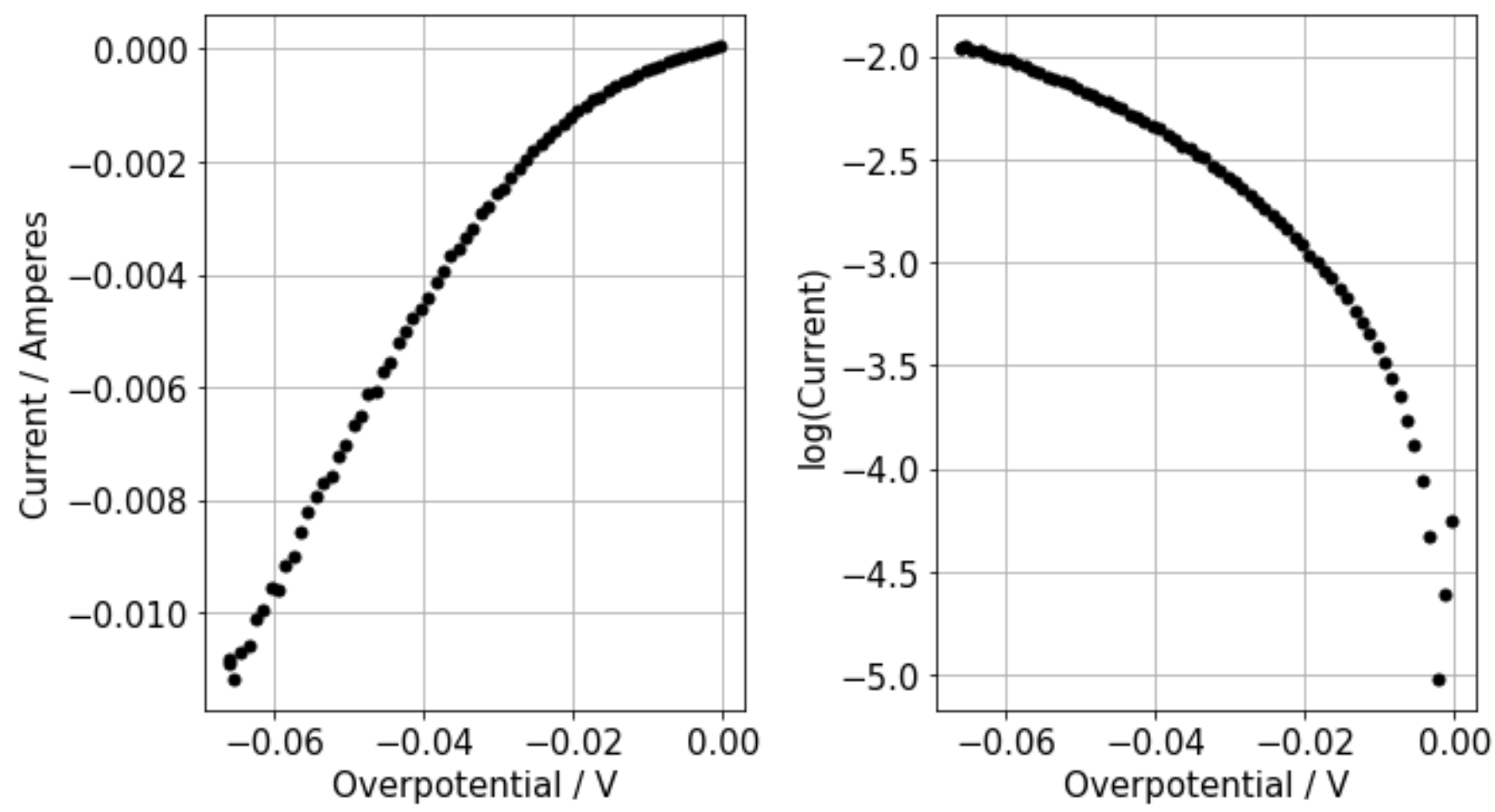

Figure S3, 1600 rpm 

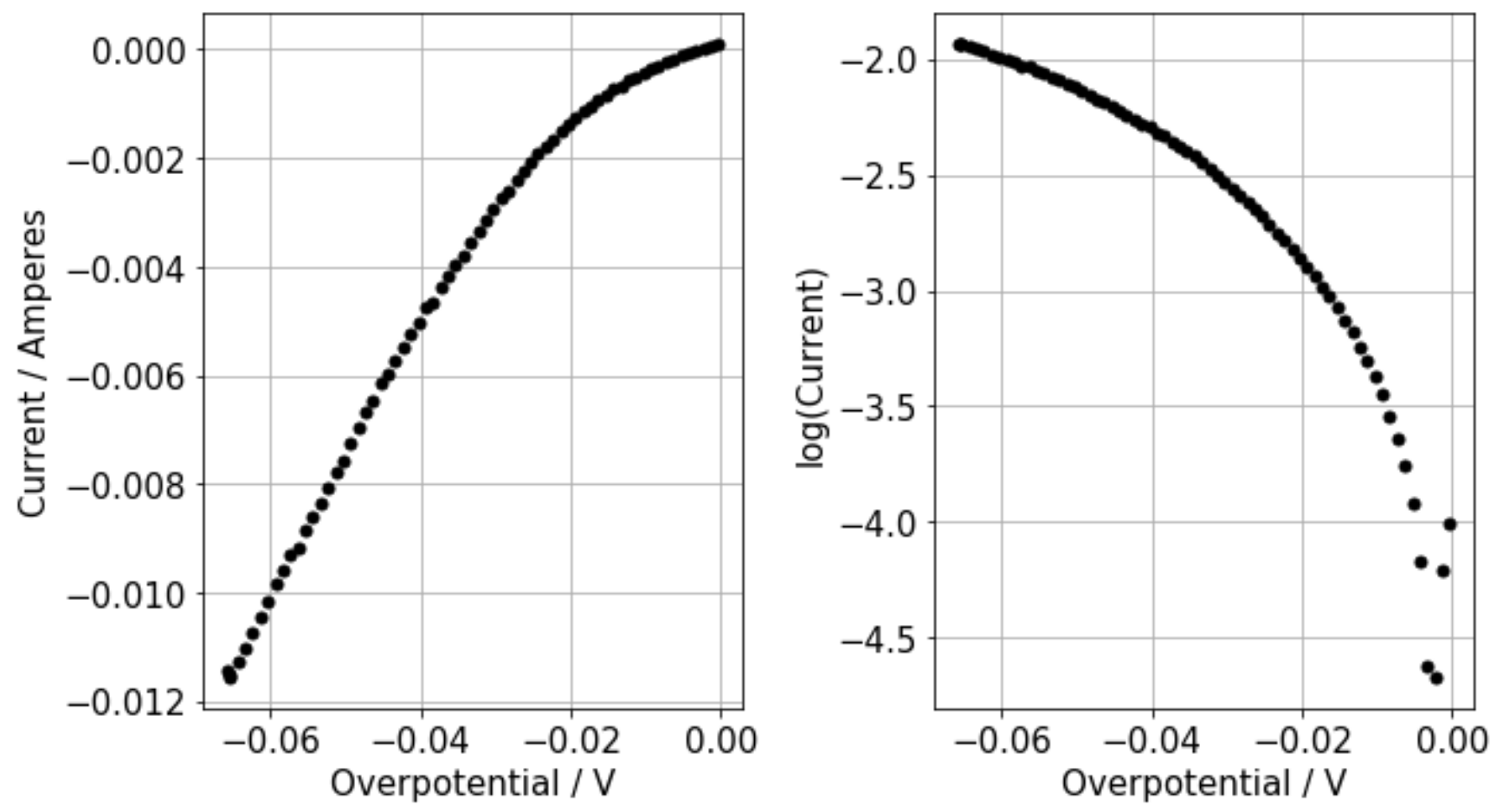

Figure S4, 2500 rpm 
Platinum-catalyzed Oxygen Reduction Reaction (ORR)

$0.1 \mathrm{M} \mathrm{HClO}_{4}$

Multiple rotation rates:
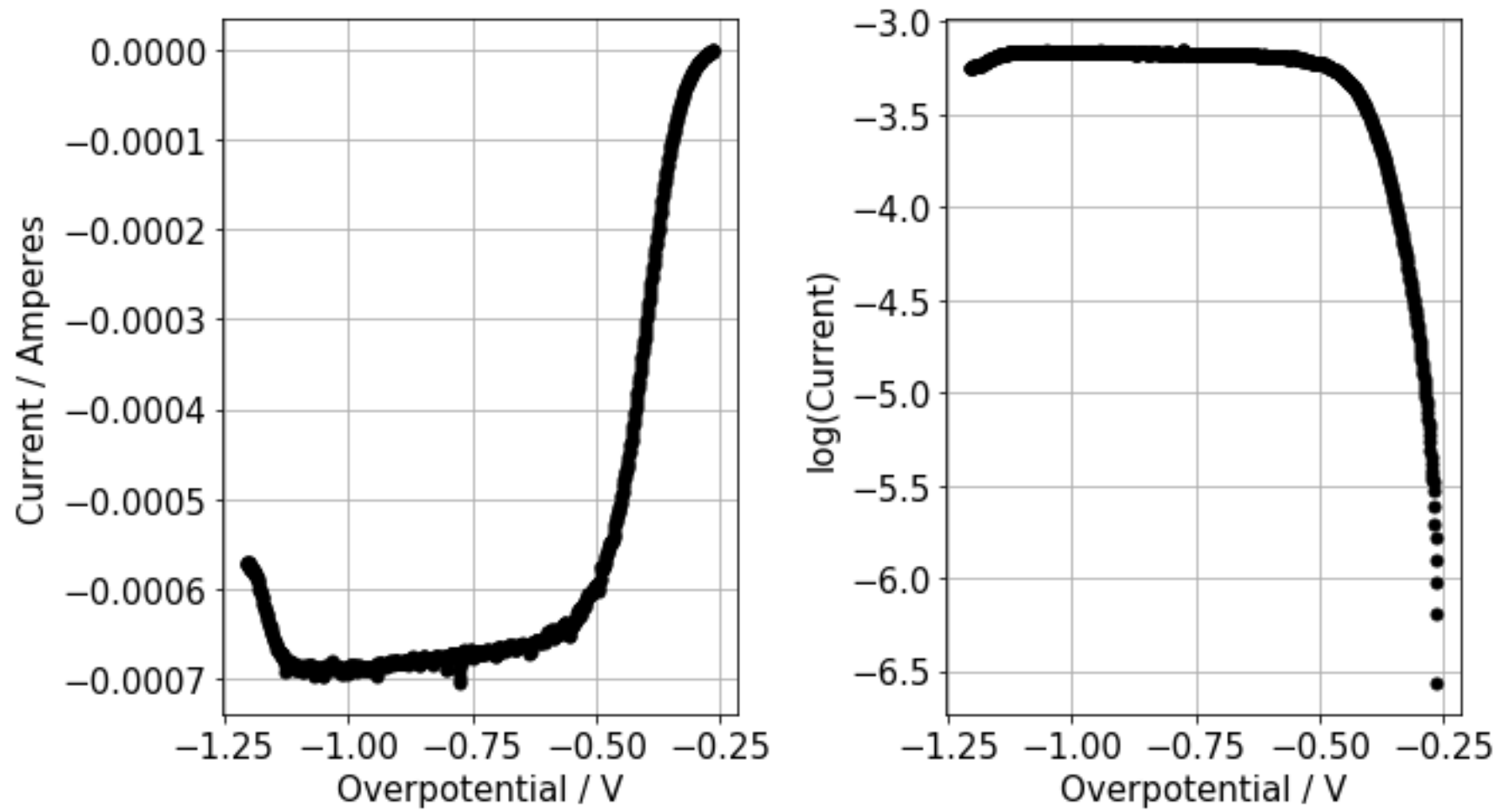

Figure S5, 900 rpm
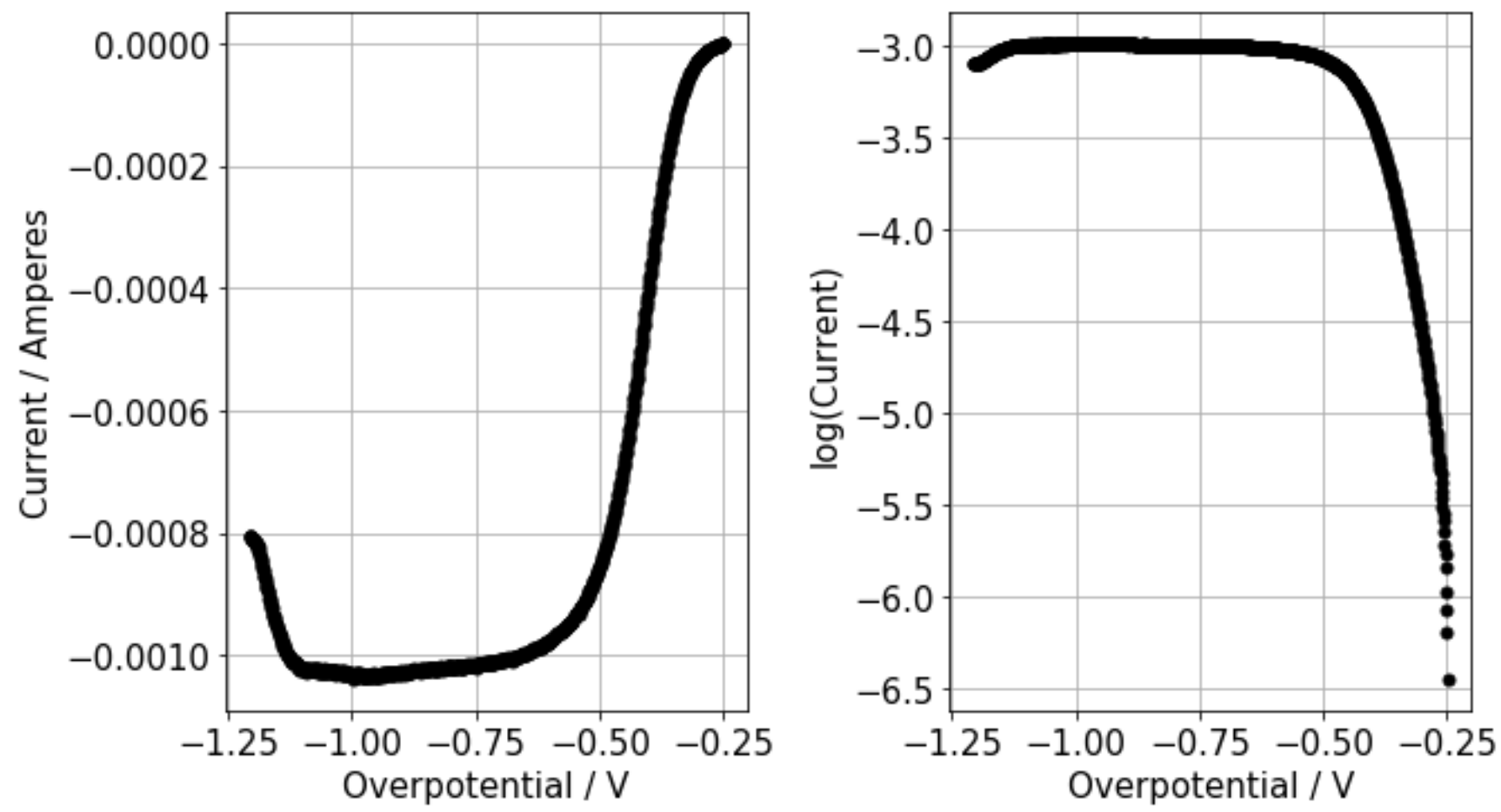

Figure S6, 1600 rpm 

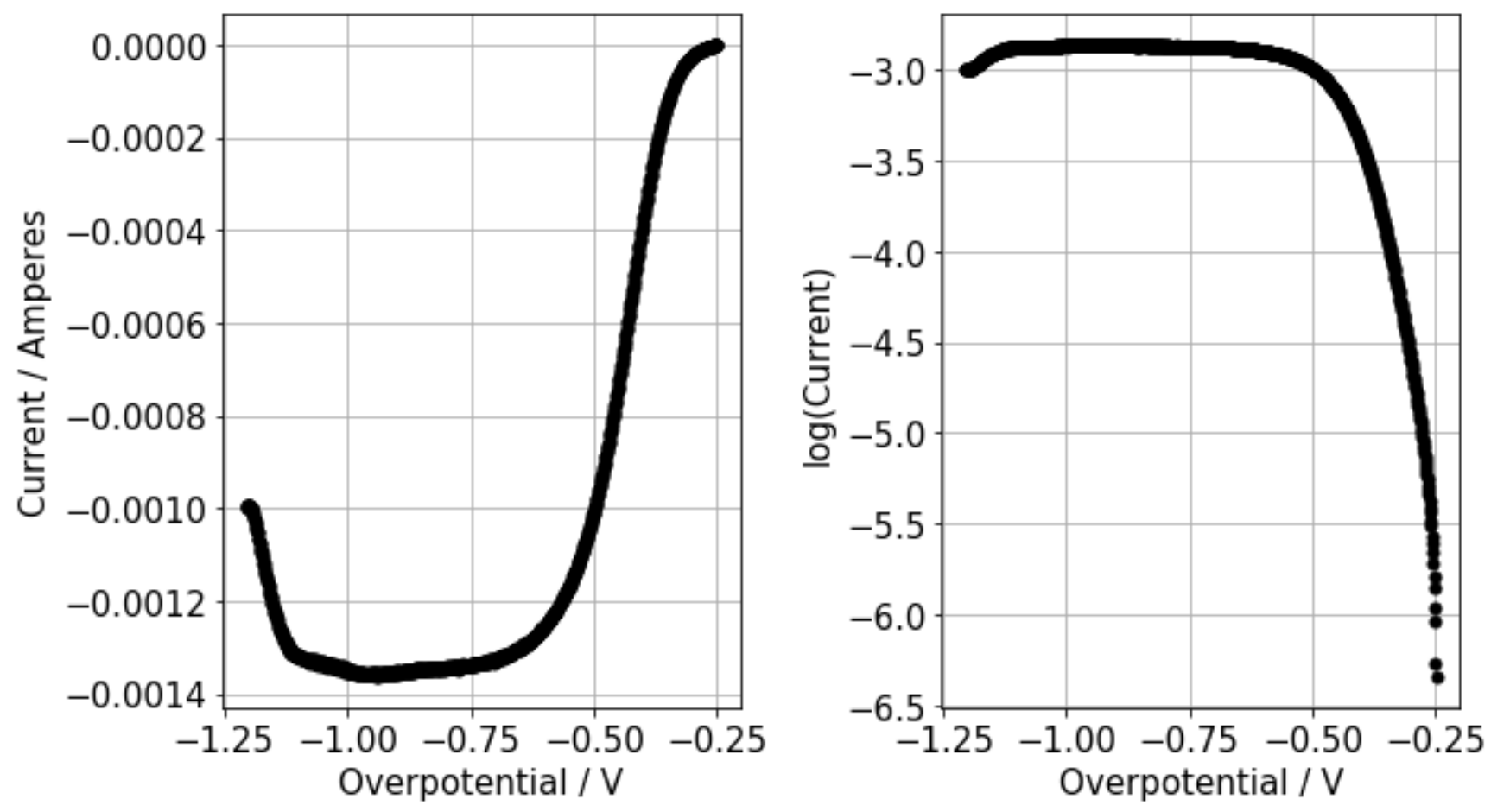

Figure S7, 2500 rpm 


\section{Program Output}

The program sweeps a dataset from high to low overpotential, performing simultaneous fits of the LSV and Tafel data according to equation (2b). For each group of fits conducted at a given $\mathrm{d} \eta$ and $\mathrm{R}^{2}$, the program produces a graphical output:
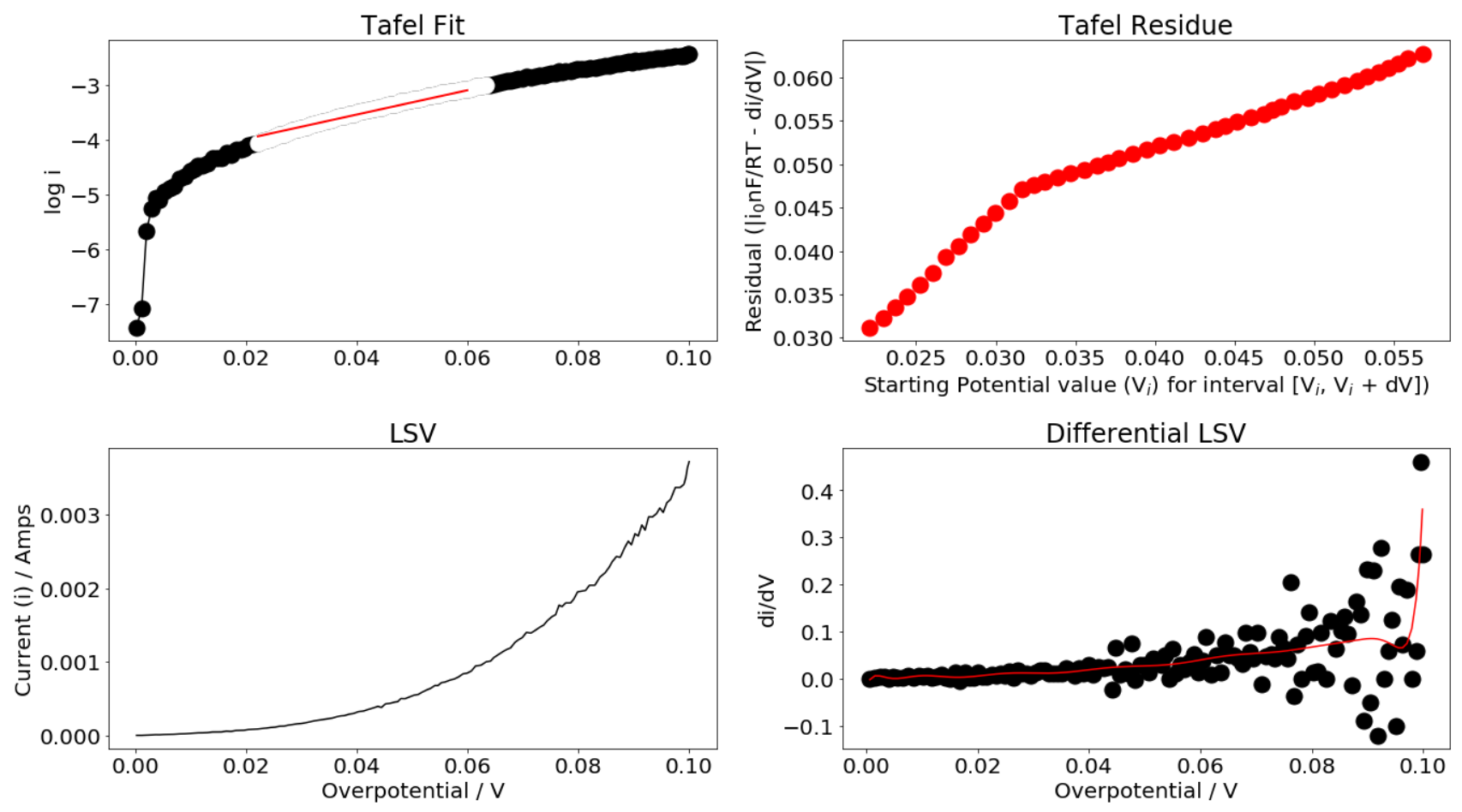

Figure S8: Fit Results file output (10/112 rows shown here) for fits satisfying the threshold pair $\mathrm{R}^{2} \geq$ $0.900, \mathrm{d \eta}=0.01$ :

$\begin{array}{cc}\text { Starting Potentials J0 } \\ 0 & 0.0028484 \\ 1 & 0.0036086 \\ 2 & 0.0043148 \\ 3 & 0.0051536 \\ 4 & 0.0060919 \\ 5 & 0.006967 \\ 6 & 0.0079662 \\ 7 & 0.0089581 \\ 8 & 0.0098116 \\ 9 & 0.0106093 \\ 10 & 0.0113062\end{array}$

\begin{tabular}{ll}
\multicolumn{2}{c}{$\mathrm{d} J / \mathrm{dV}$} \\
$4.39 \mathrm{E}-06$ & 0.003512646644258 \\
$5.31 \mathrm{E}-06$ & 0.003723896956107 \\
$5.92 \mathrm{E}-06$ & 0.003951239641122 \\
$7.17 \mathrm{E}-06$ & 0.004125777631577 \\
$8.01 \mathrm{E}-06$ & 0.004476880356497 \\
$9.12 \mathrm{E}-06$ & 0.004765673887045 \\
$1.04 \mathrm{E}-05$ & 0.005103581407877 \\
$1.12 \mathrm{E}-05$ & 0.005555711959537 \\
$1.21 \mathrm{E}-05$ & 0.005993403736046 \\
$1.26 \mathrm{E}-05$ & 0.006560136495623 \\
$1.32 \mathrm{E}-05$ & 0.007069771190336
\end{tabular}

Tafel Slope / $\mathrm{mV}$ decade-1 13.3795863455284 15.2220023358312 16.5434835627867 18.7073017591735 20.0917032519155 21.8240920311893 23.6558151586603 24.68324502755 25.8511231890353 26.4537118667183 27.1602793584385

Fit Summary file output for the fit in the fit results array that has the lowest Tafel residue:

$$
\begin{array}{crrrr}
\text { Bound 1 }(\mathrm{V}) & \text { Bound 2 }(\mathrm{V}) & \text { Exchange Current } & \multicolumn{2}{c}{\text { Tafel Slope }} \\
0 & 0.0028484 & 0.0128484 & 4.39 \mathrm{E}-06 & 13.3795863455284
\end{array}
$$

Table S1 
After all possible fits for the range of user-specified dns and $\mathrm{R}^{2} \mathrm{~s}$ have been tested, and the fit containing the smallest Tafel residue for any particular $d \eta / R^{2}$ pair are stored in an array. This array represents the set of residue-minimized fits for the particular polarization curve being fit, over the range of dns and $\mathrm{R}^{2} \mathrm{~s}$ specified. From this residue-minimized set, dn-span and $\mathrm{R}^{2}$ optimizations proceed, and yield the following type of chart output, along with the primary visualization output:

\begin{tabular}{llrrrrr}
\multicolumn{2}{c}{ Tafel Bin / mV decade- } & R2 threshold & Overpotential Width / V & Tafel slope / mV Decade- 1 & Exchange Current / A & Optimal Residual \\
0 & 64.0043472666134 & 0.976 & 0.045 & 63.2361694888095 & $9.99 \mathrm{E}-05$ & 0.059952045721865 \\
1 & 64.0043472666134 & 0.976 & 0.04 & 63.2361694888095 & $9.99 \mathrm{E}-05$ & 0.059952045721865 \\
2 & 64.0043472666134 & 0.976 & 0.035 & 63.2361694888095 & $9.99 \mathrm{E}-05$ & 0.059952045721865 \\
3 & 64.0043472666134 & 0.976 & 0.03 & 63.2361694888095 & $9.99 \mathrm{E}-05$ & 0.059952045721865 \\
4 & 64.0043472666134 & 0.976 & 0.02 & 63.2361694888095 & $9.99 \mathrm{E}-05$ & 0.059952045721865 \\
5 & 64.0043472666134 & 0.976 & 0.015 & 63.2361694888095 & $9.99 \mathrm{E}-05$ & 0.059952045721865 \\
6 & 64.0043472666134 & 0.976 & 0.025 & 63.2361694888095 & $9.99 \mathrm{E}-05$ & 0.059952045721865
\end{tabular}

Starting Fit Overpotential / V Tafel R2 LSV R2 $0.0540810 .993938091903666 \quad 0.976683149583044$ $0.054081 \quad 0.993938091903666 \quad 0.976683149583044$ $0.054081 \quad 0.993938091903666 \quad 0.976683149583044$ $0.054081 \quad 0.993938091903666 \quad 0.976683149583044$ $0.054081 \quad 0.993938091903666 \quad 0.976683149583044$ $\begin{array}{lll}0.054081 & 0.993938091903666 & 0.976683149583044\end{array}$ $\begin{array}{lll}0.054081 & 0.993938091903666 & 0.976683149583044\end{array}$

\section{Table S2}

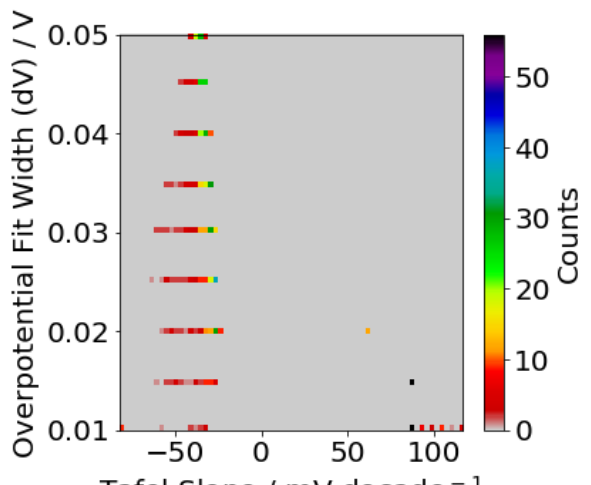

Tafel Slope / $\mathrm{mV}$ decade ${ }^{-1}$

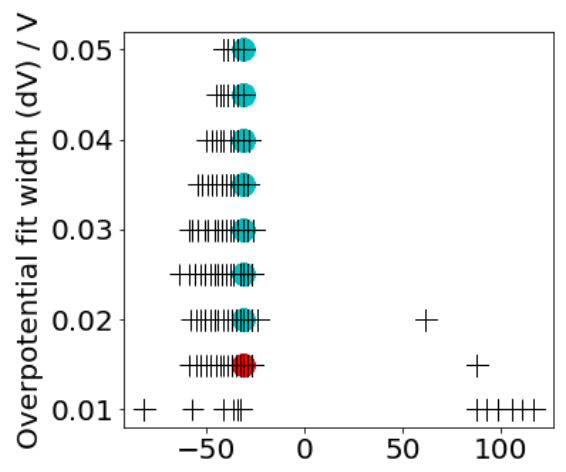

Tafel Slope Bin / $\mathrm{mV}$ decade ${ }^{-1}$
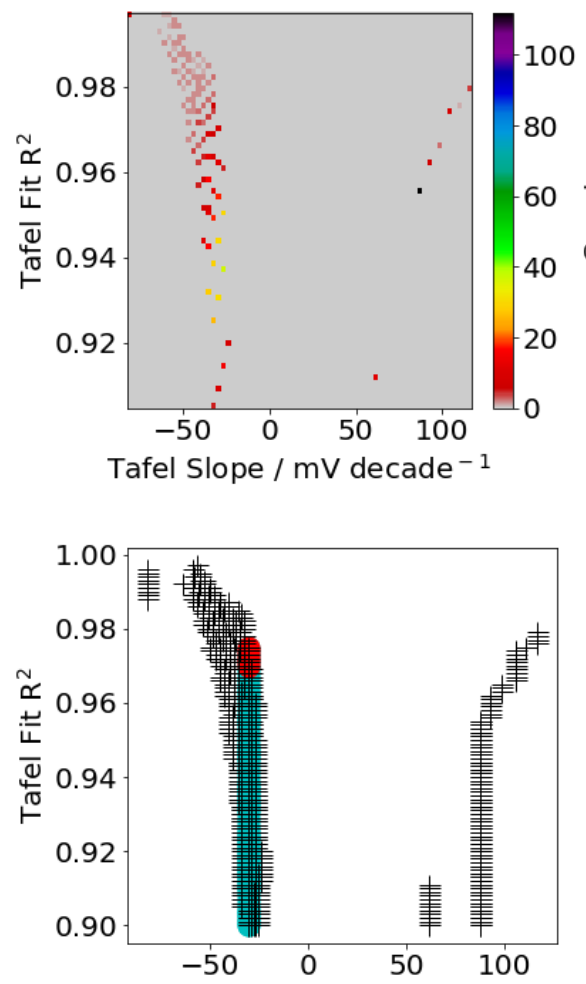

Tafel Slope Bin / mV decade ${ }^{-1}$
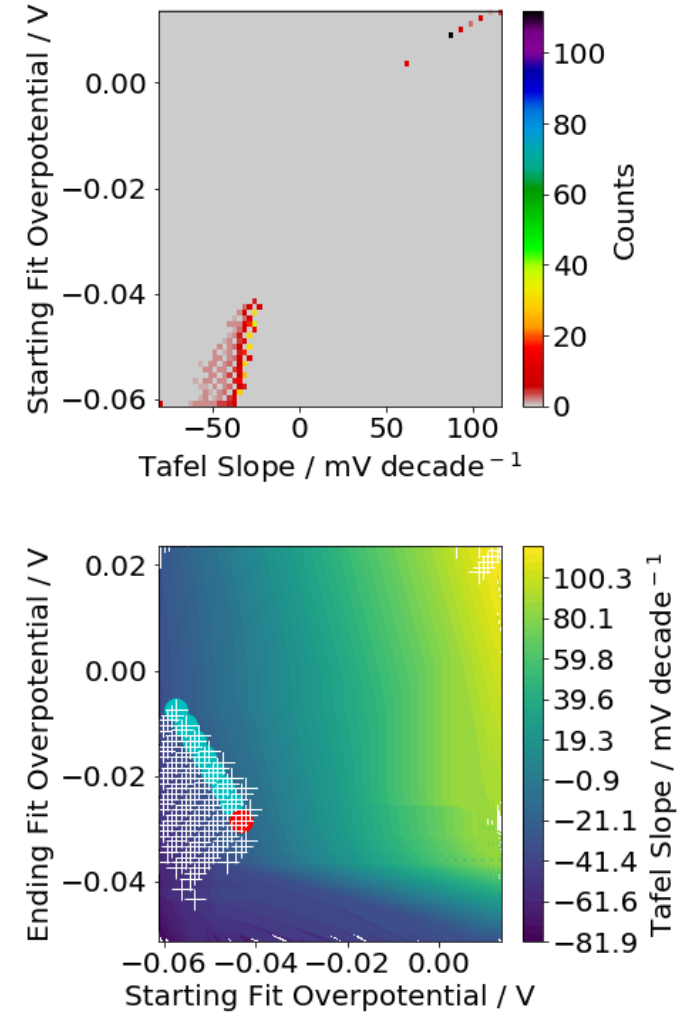

Figure S9 
Influence of $\mathbf{R}^{2}$ Thresholds - Ir OER Data examples, T= 298K, Binned at $1 \mathrm{mV}$
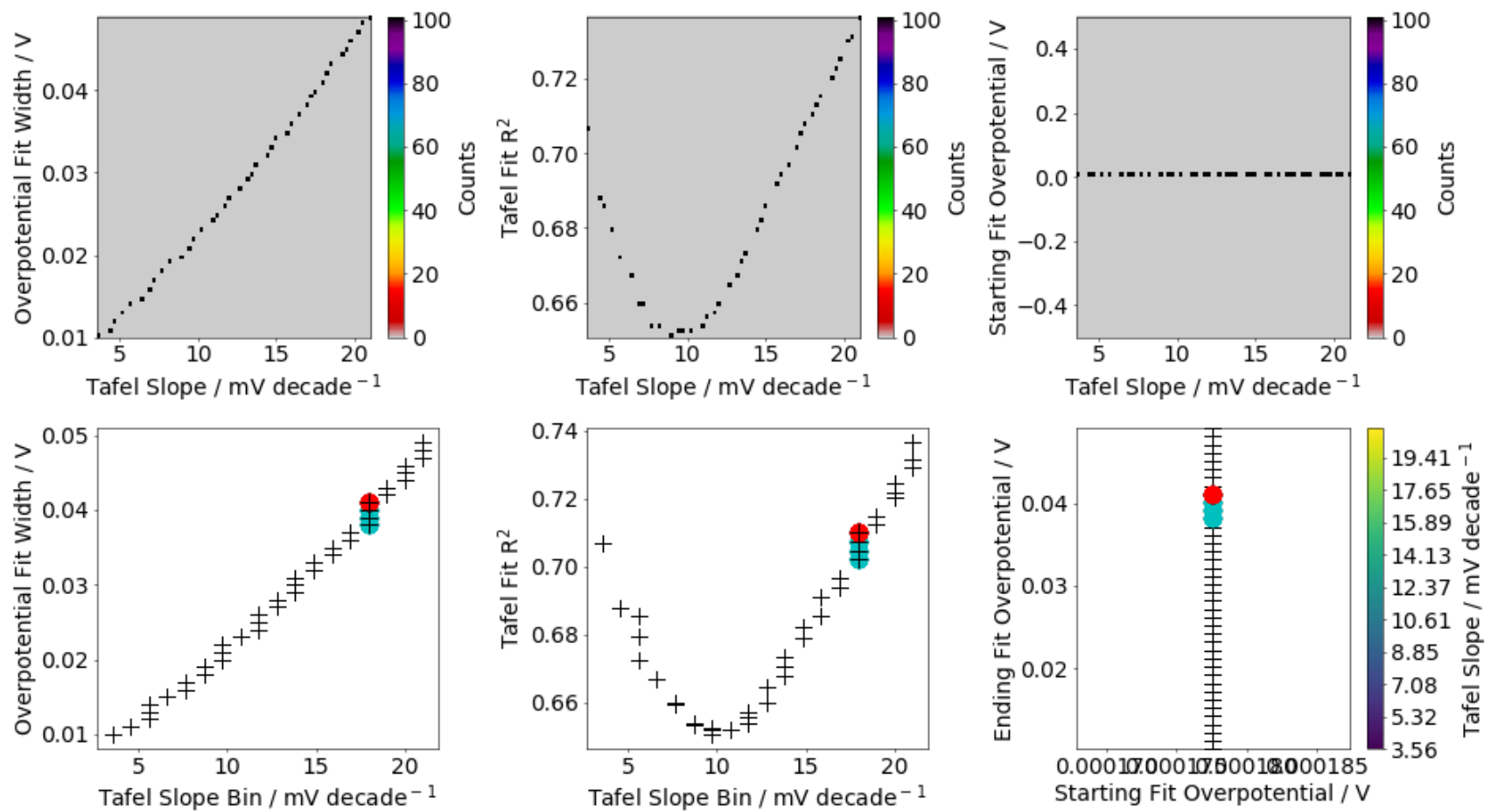

Figure S10: $\mathrm{d} \eta=10-50 \mathrm{mV}, 1 \mathrm{mV}$ increments; $\mathrm{R}^{2}=\mathbf{0 . 5 0 0 - 0 . 6 0 0}, 0.001$ increments; optimal fit = 18 mV decade $^{-1}$
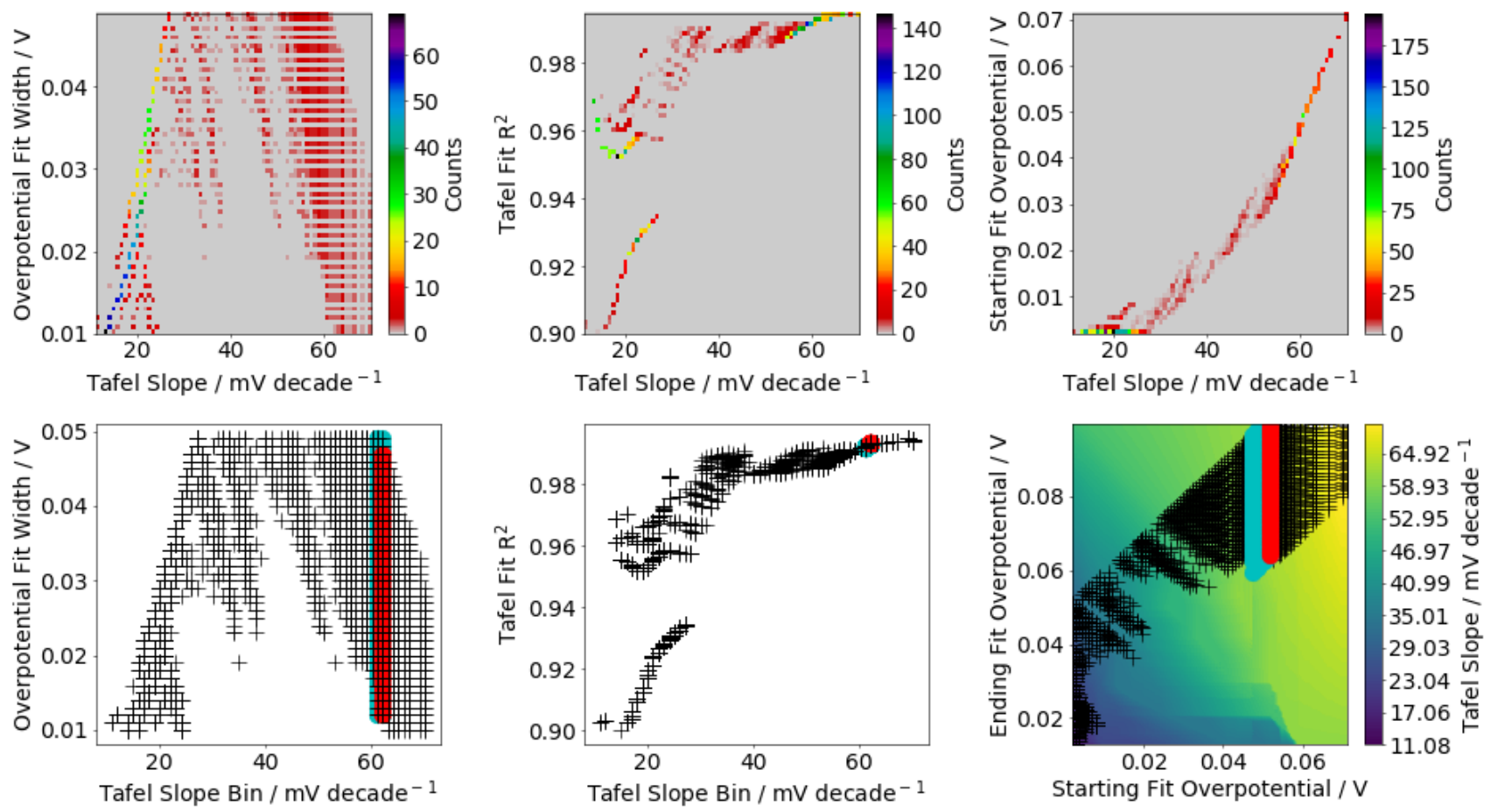

Figure S11: $\mathrm{d} \eta=10-50 \mathrm{mV}, 1 \mathrm{mV}$ increments; $\mathrm{R}^{2}=\mathbf{0 . 9 0 0 - 1 . 0 0 0}, 0.001$ increments; optimal fit = 62 mV decade $^{-1}$ 


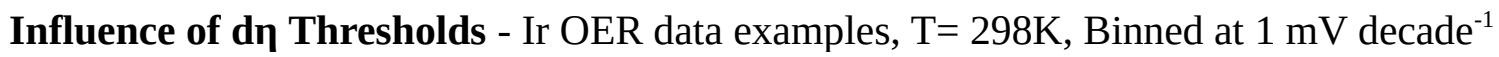

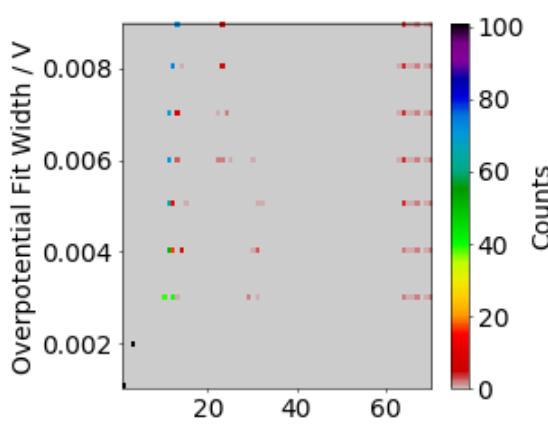

Tafel Slope / $\mathrm{mV}$ decade -1

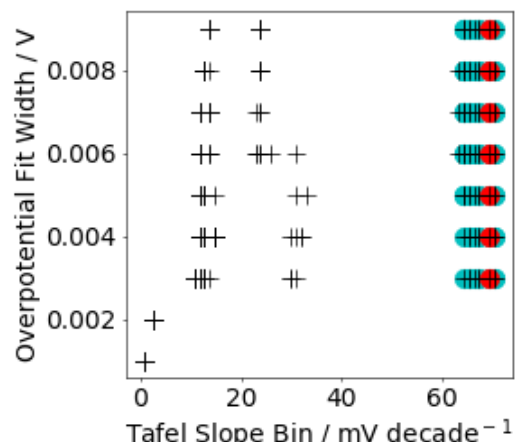

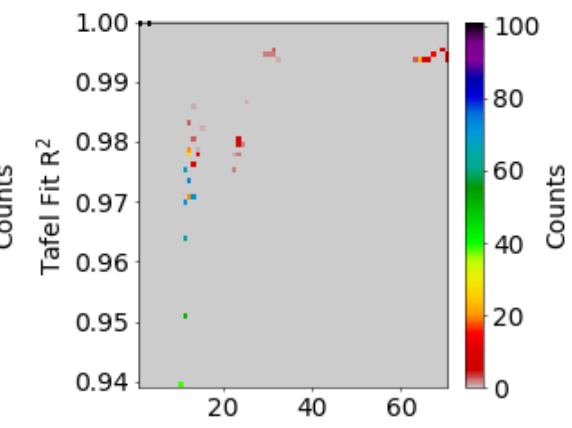

Tafel Slope / $\mathrm{mV}$ decade ${ }^{-1}$

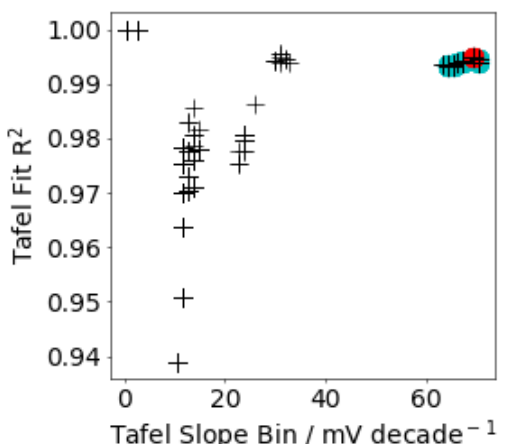

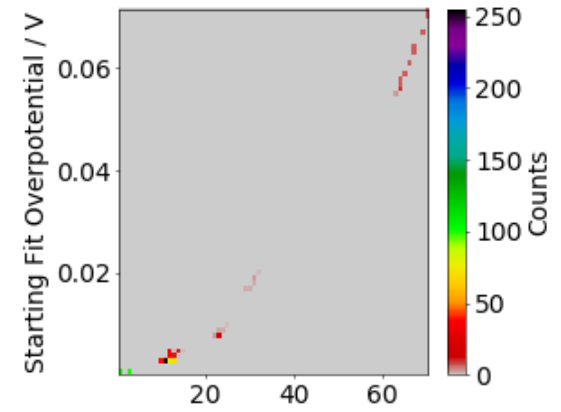

Tafel Slope / $\mathrm{mV}$ decade -1

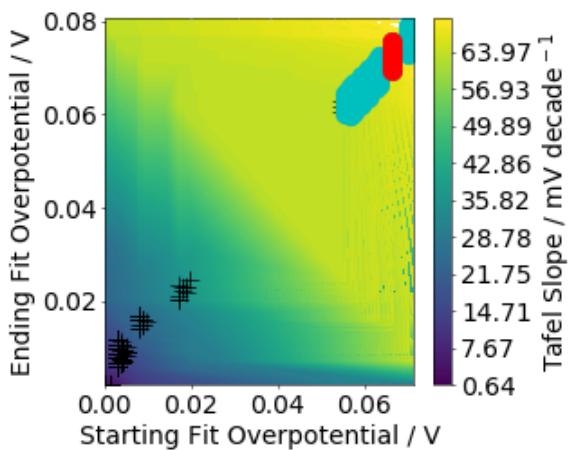

Figure S12: $\mathrm{d} \eta=\mathbf{1 - 1 0} \mathrm{mV}, 1 \mathrm{mV}$ increments; $\mathrm{R}^{2}=0.900-1.000,0.001$ increments; optimal fit = $68 \mathbf{~ m V}$ decade $^{-1}$
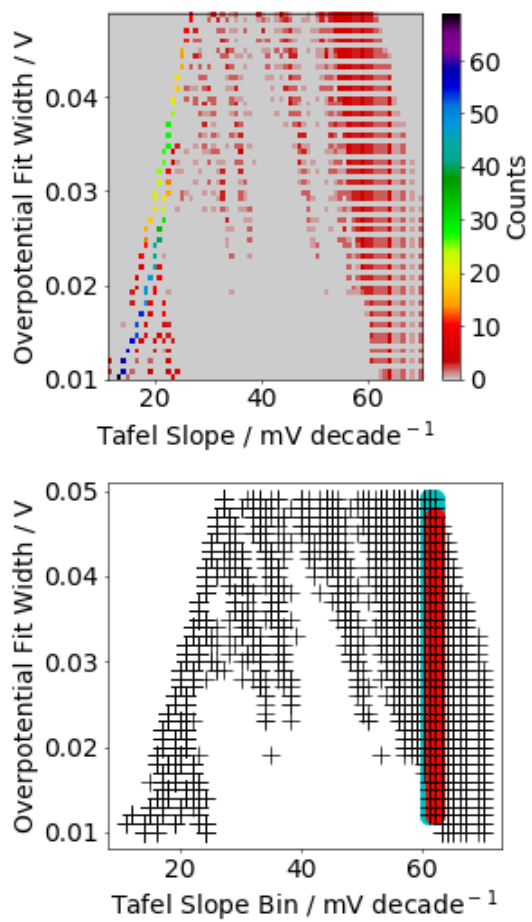
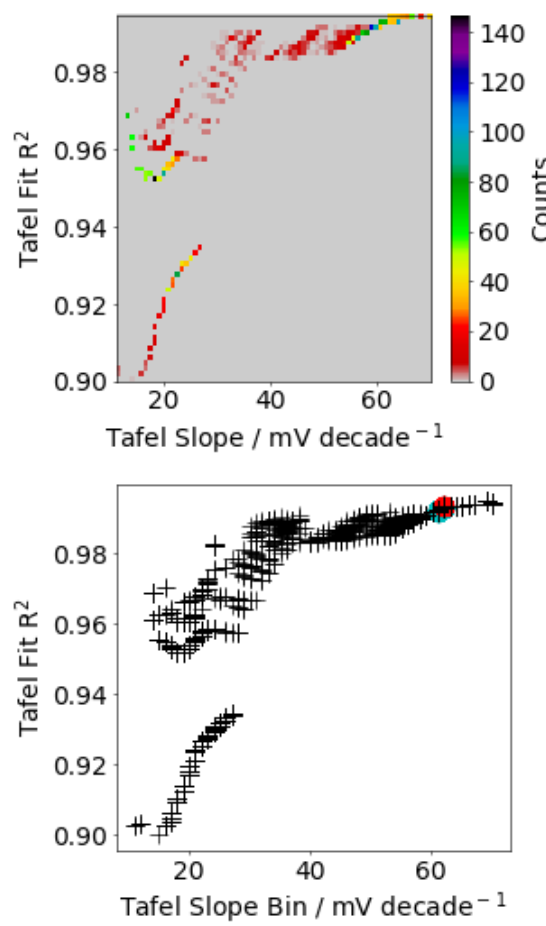
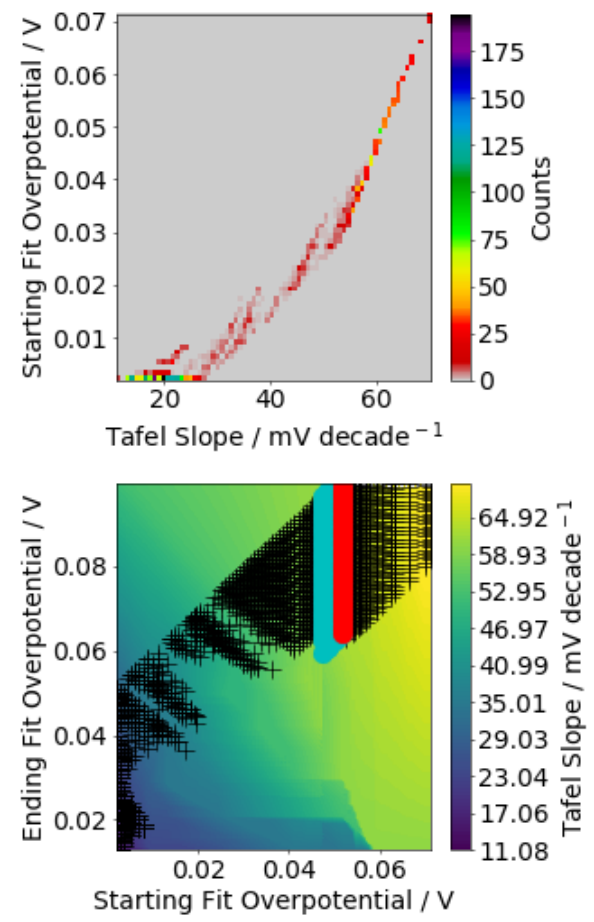

Figure S13: $\mathrm{d} \eta=\mathbf{1 0}-\mathbf{5 0} \mathbf{~ m V}, 1 \mathrm{mV}$ increments; $\mathrm{R}^{2}=0.900-1.000,0.001$ increments; optimal fit = 62 mV decade $^{-1}$ 
Influence of Binning - Ir OER data examples, $\mathrm{T}=293 \mathrm{~K}, \mathrm{d \eta}=10$ to $50 \mathrm{mV}, 1 \mathrm{mV}$ increments, $\mathrm{R}^{2}=$ 0.900-1.000, 0.001 increments
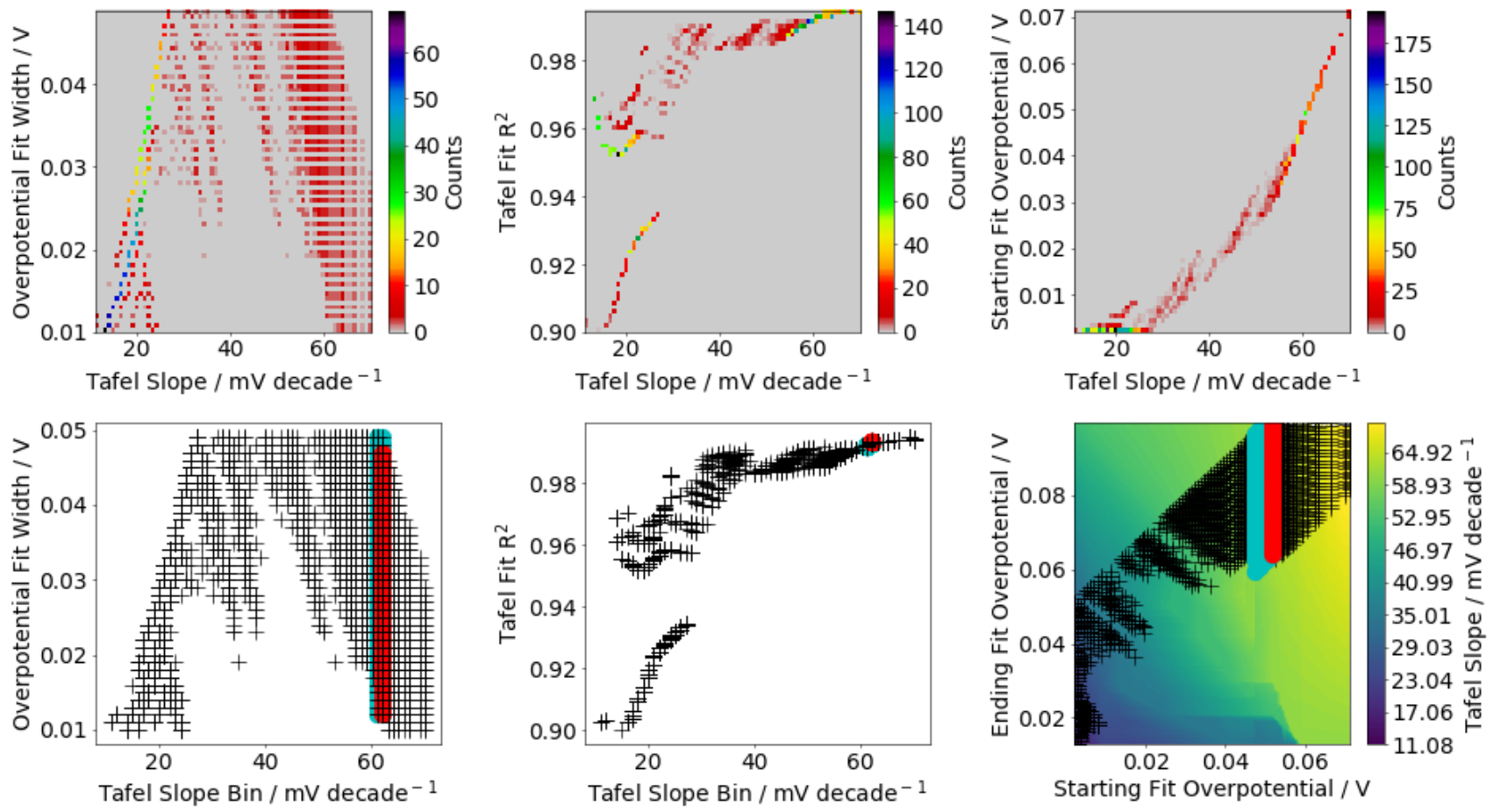

Figure S14: Binning = $1 \mathbf{~ m V ~ d e c a d e ~}{ }^{-1}$; optimal fit $=62 \mathrm{mV}$ decade $^{-1}$
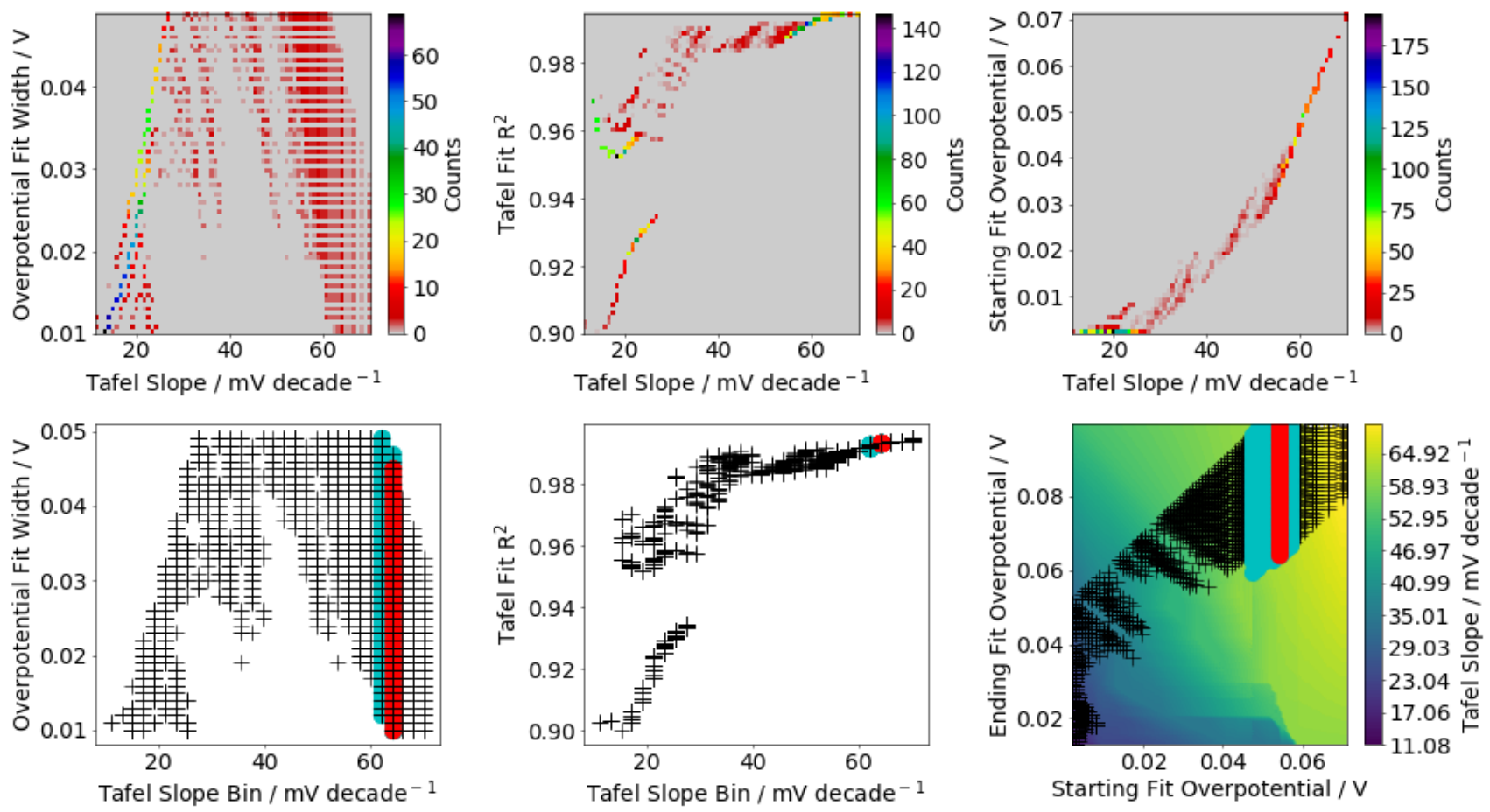

Figure S15: Binning = $2 \mathbf{m V}$ decade ${ }^{-1}$; optimal fit $=63 \mathrm{mV}$ decade $^{-1}$ 

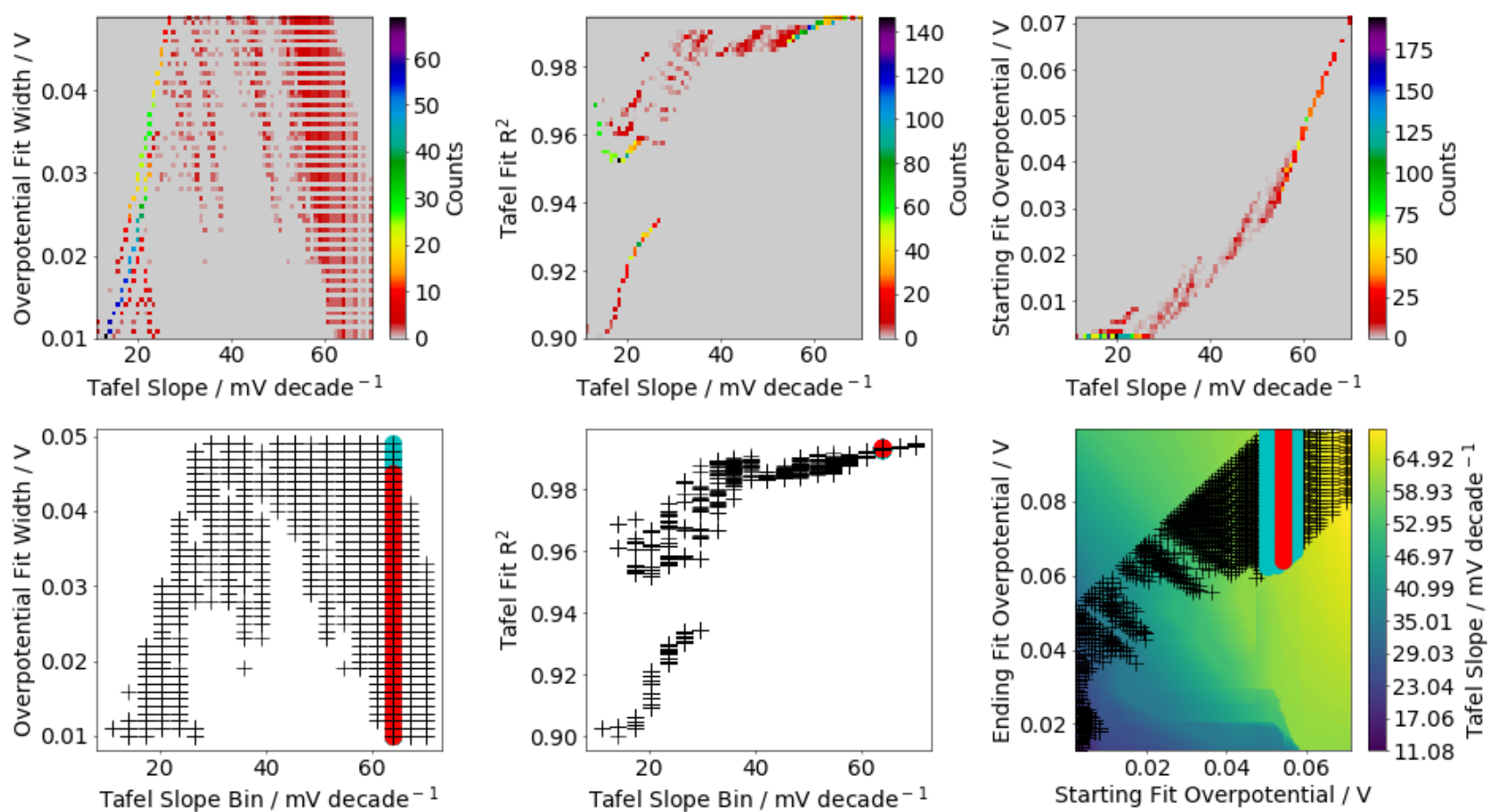

Figure S16: Binning = $\mathbf{3} \mathbf{~ m V ~ d e c a d e ~}{ }^{-1}$; optimal fit $=63 \mathrm{mV}$ decade $^{-1}$
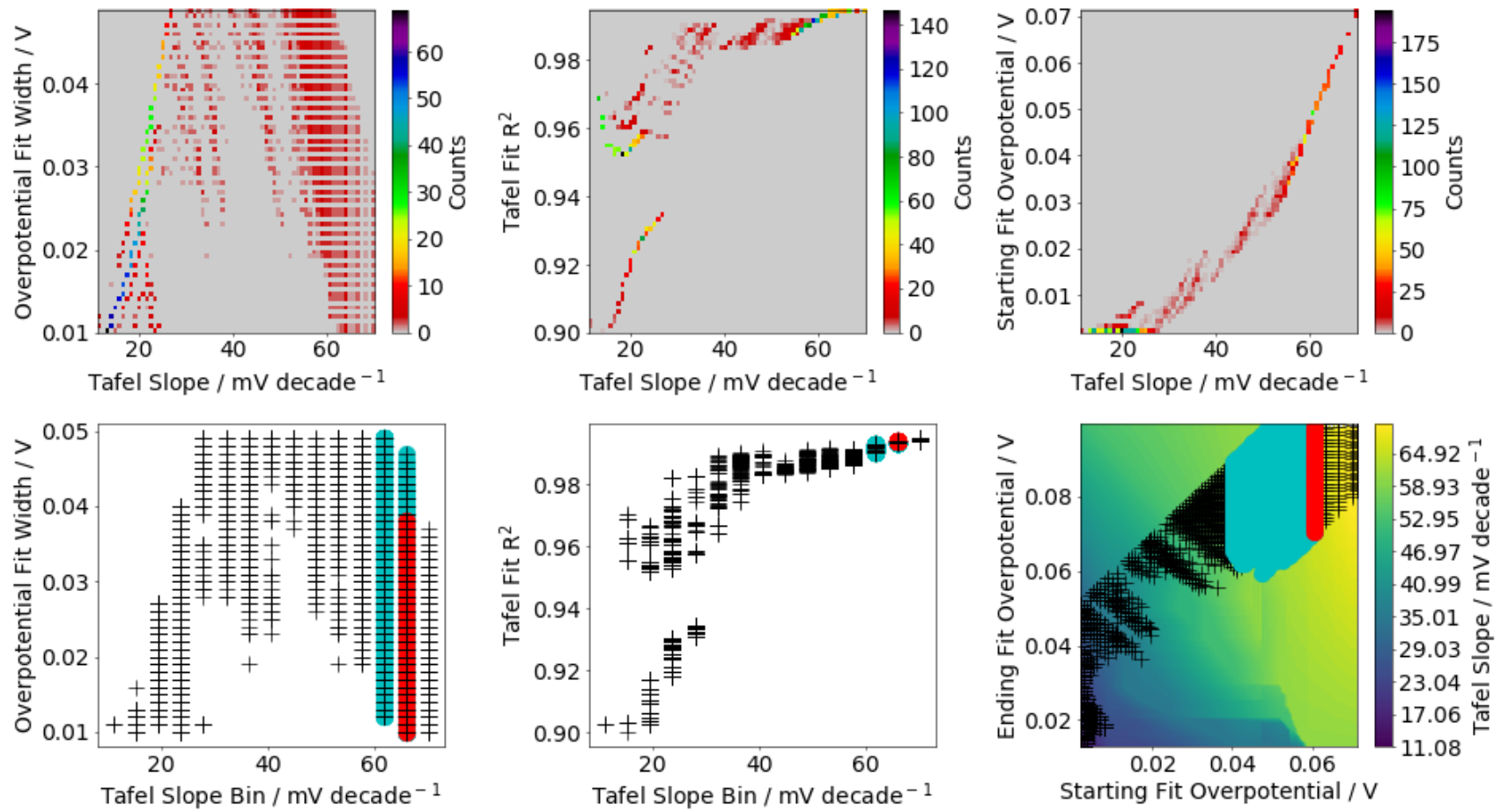

Figure S17: Binning $=\mathbf{4} \mathbf{~ m V ~ d e c a d e ~}{ }^{-1}$; optimal fit $=66 \mathrm{mV}$ decade $^{-1}$ 

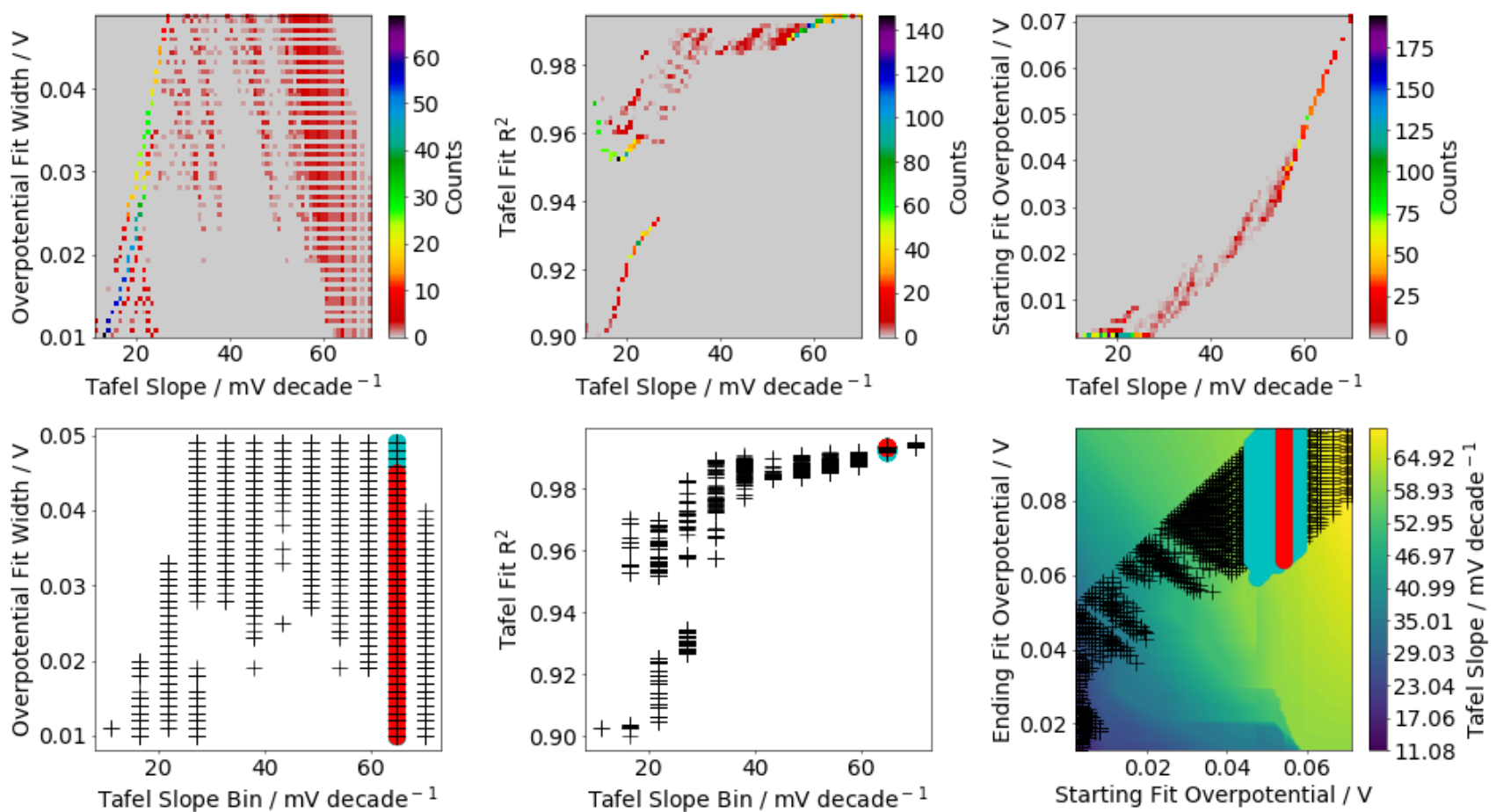

Figure S18: Binning $=\mathbf{5} \mathbf{~ m V ~}$ decade $^{-1}$; optimal fit $=63 \mathrm{mV}$ decade -1
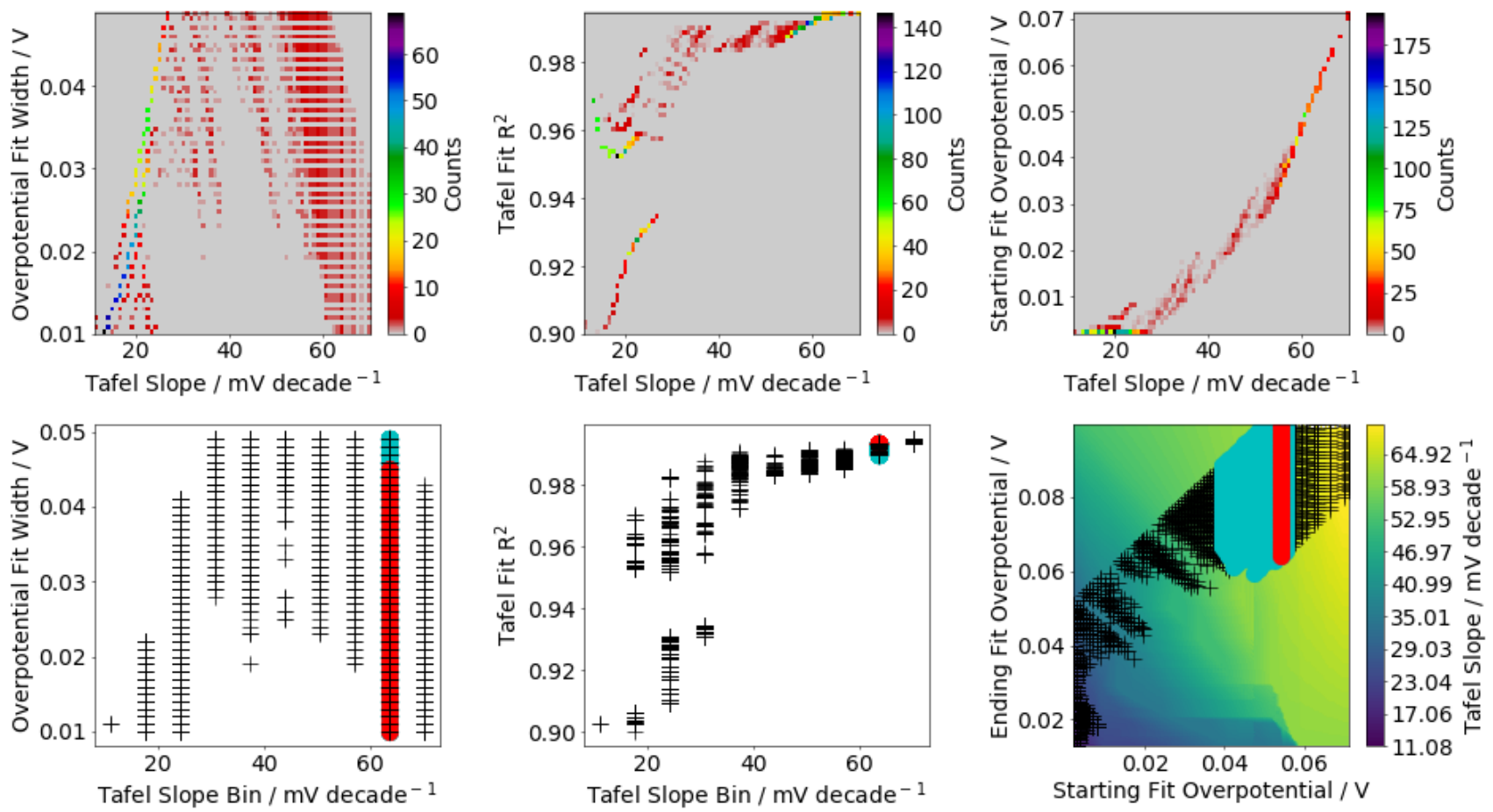

Figure S19: Binning $=\mathbf{6} \mathbf{~ m V}$ decade $^{-1}$; optimal fit $=63 \mathrm{mV}$ decade $^{-1}$ 

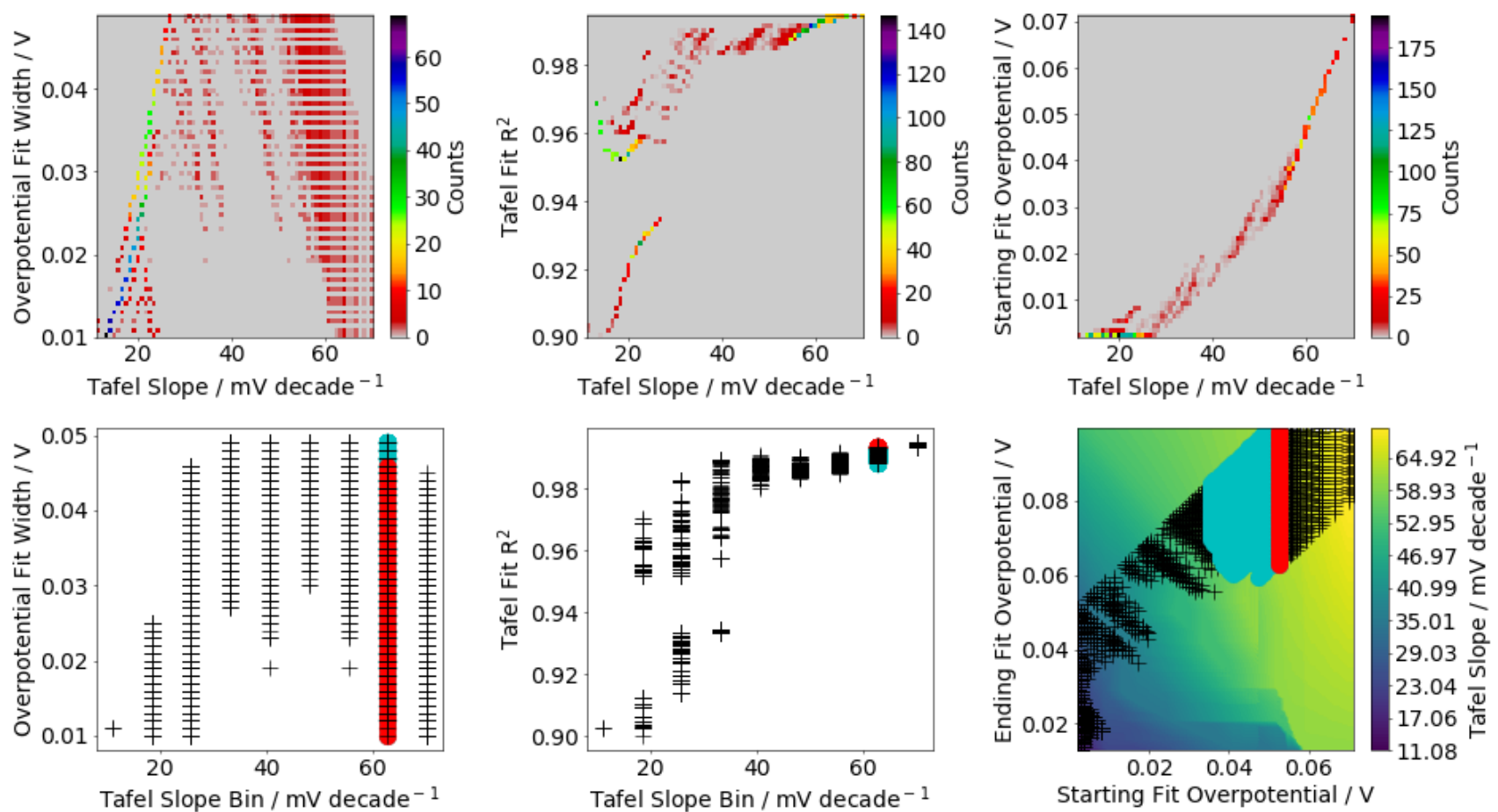

Figure S20: Binning $=7 \mathbf{~ m V ~ d e c a d e ~}{ }^{-1}$; optimal fit $=62 \mathrm{mV}$ decade $^{-1}$
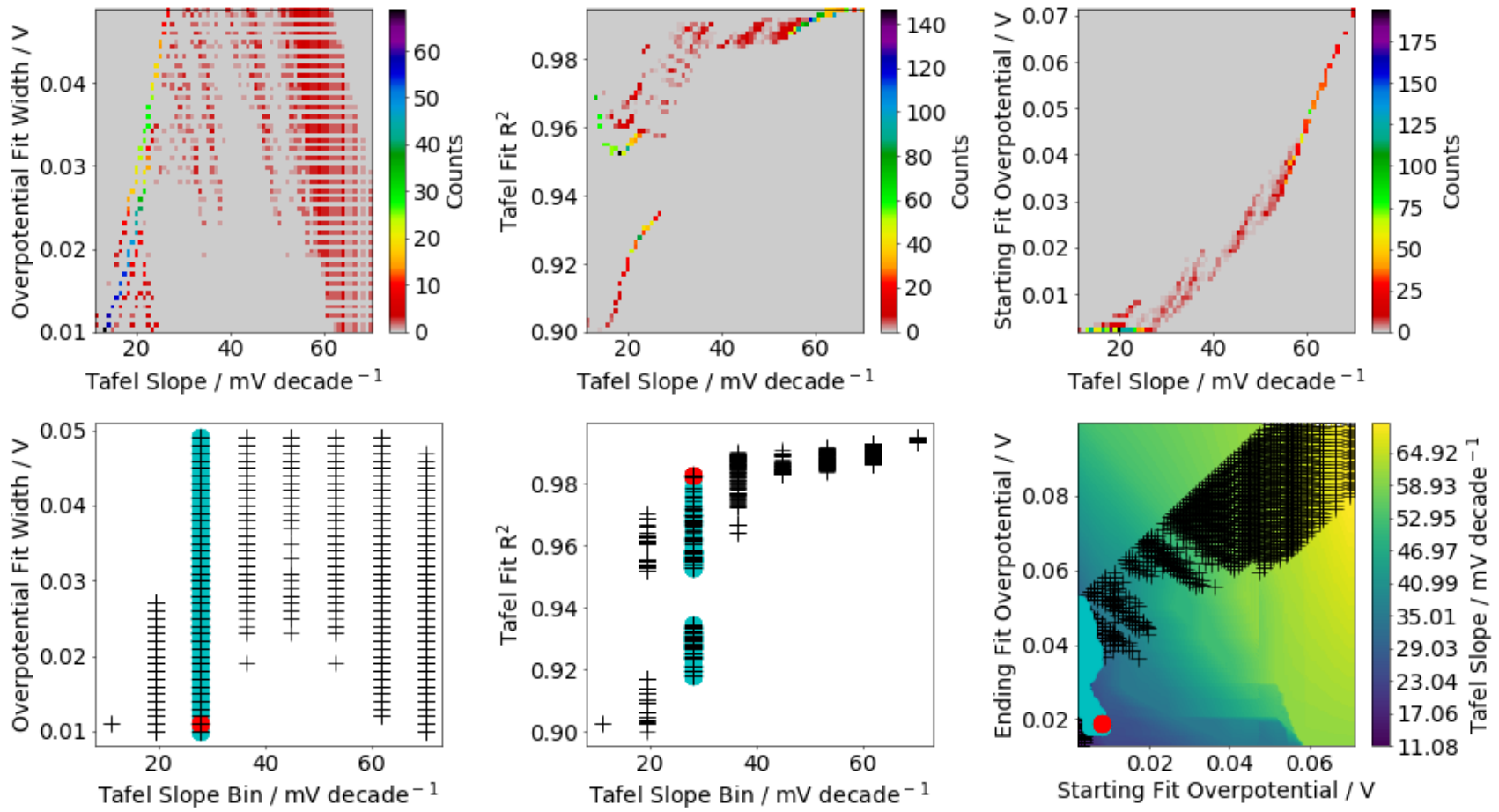

Figure S21: Binning $=\mathbf{8} \mathbf{~ m V ~ d e c a d e ~}{ }^{-1}$; optimal fit $=24 \mathrm{mV}_{\text {decade }}{ }^{-1}$ 

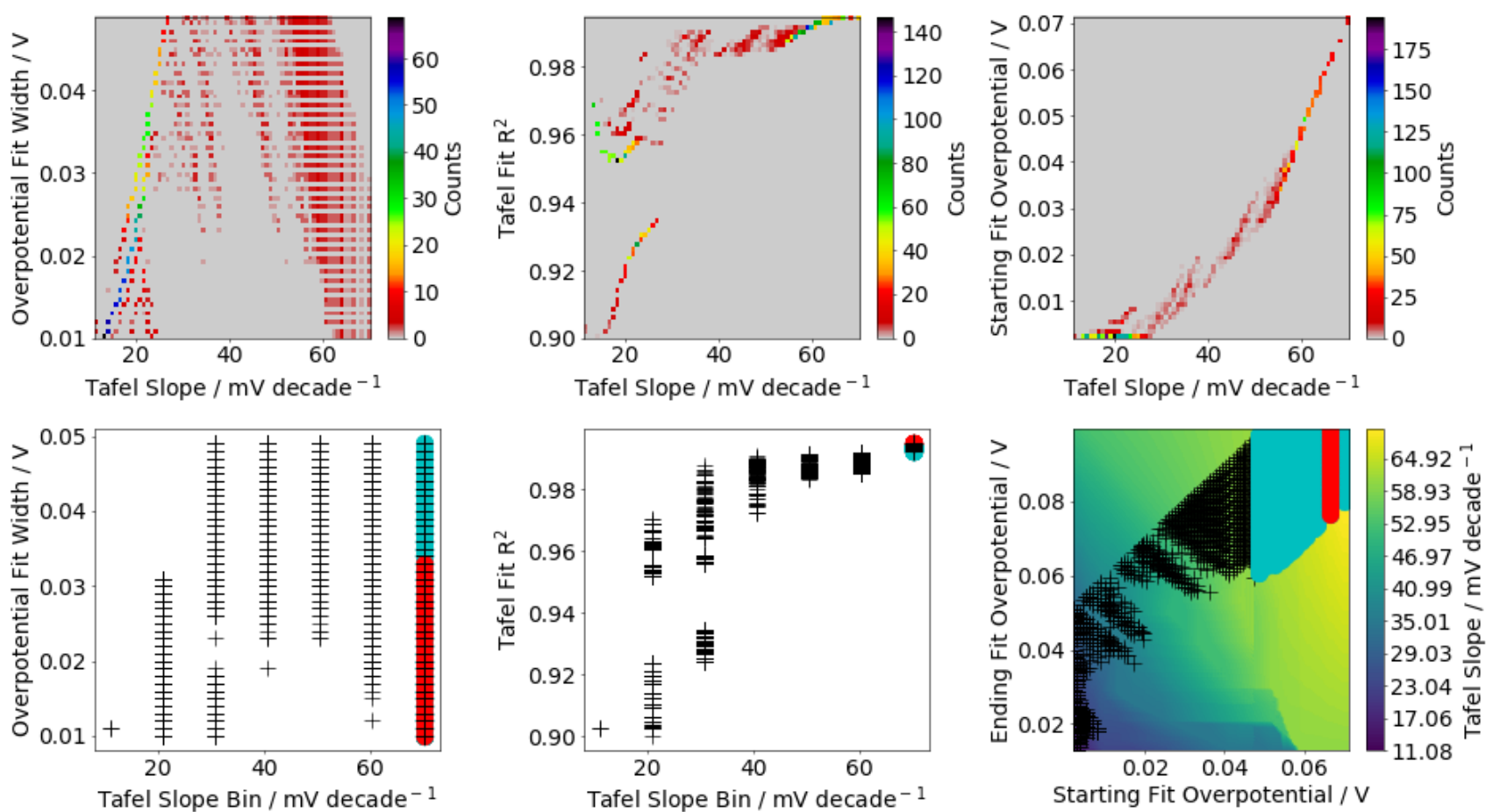

Figure S22: Binning $=\mathbf{9} \mathbf{~ m V ~ d e c a d e ~}{ }^{-1}$; optimal fit $=68 \mathrm{mV}$ decade $^{-1}$
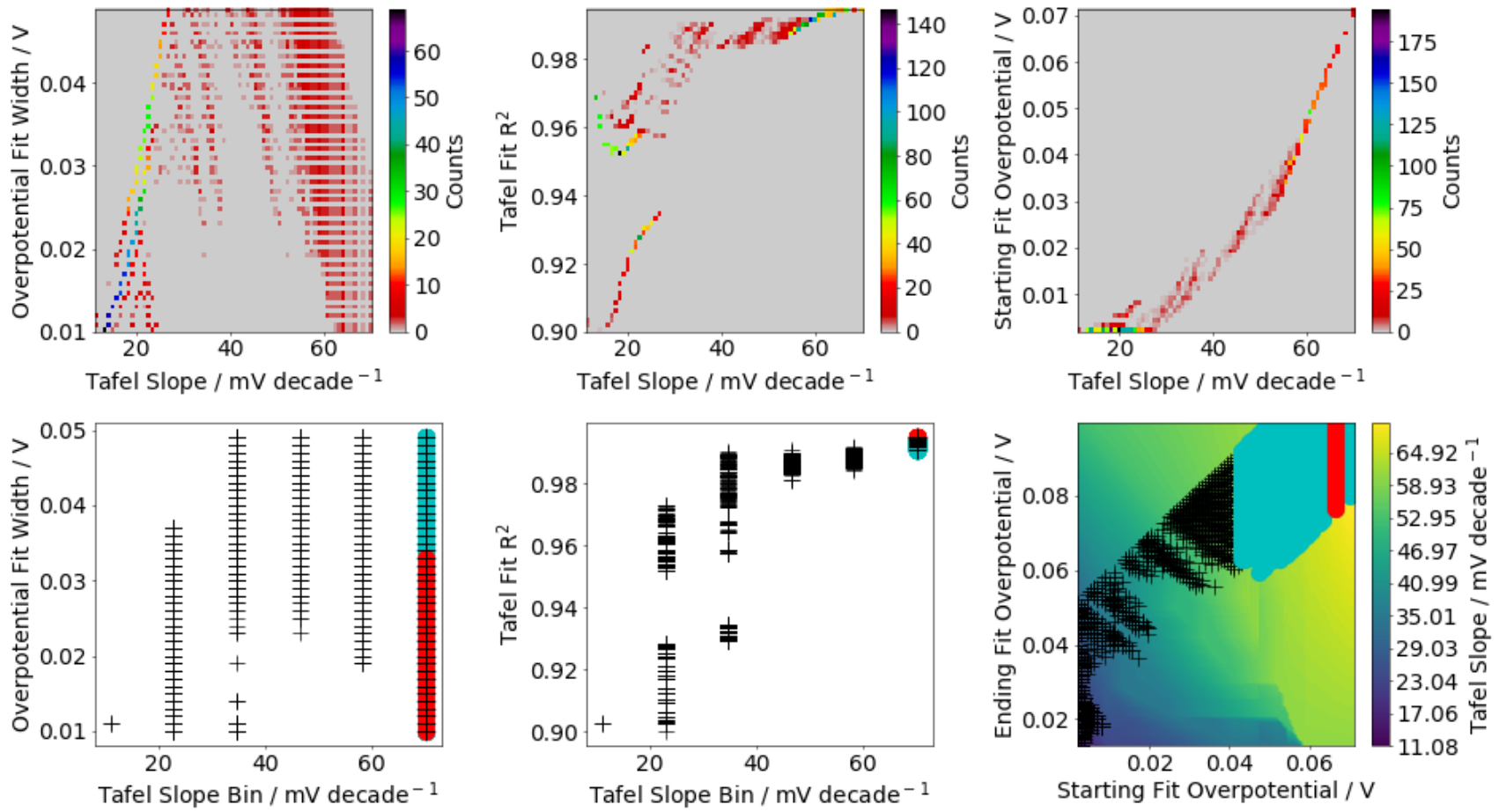

Figure S23: Binning $=\mathbf{1 0 ~} \mathbf{~ m V ~ d e c a d e ~}{ }^{-1}$; optimal fit $=68 \mathrm{mV}$ decade $^{-1}$ 
HER and ORR Fits at 900 and 1600 rpm, Binning $=1 \mathrm{mV}$ decade $^{-1}$

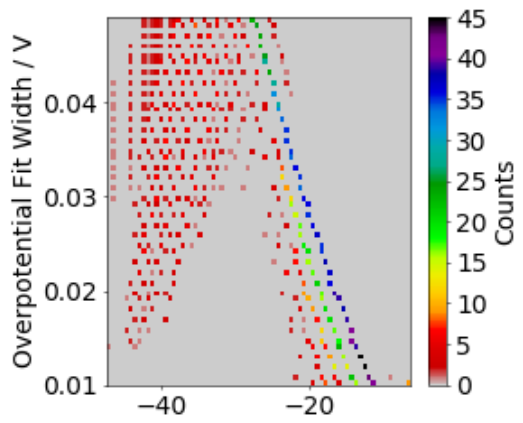

Tafel Slope / $\mathrm{mV}$ decade ${ }^{-1}$

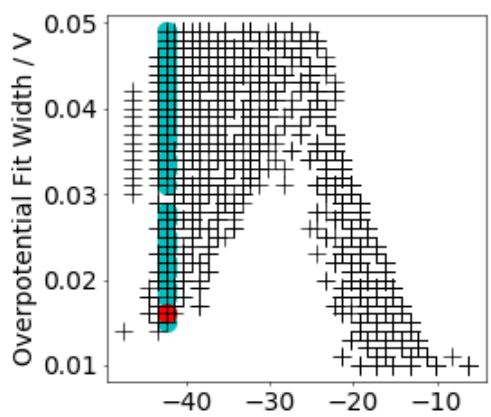

Tafel Slope Bin / $\mathrm{mV}$ decade ${ }^{-1}$

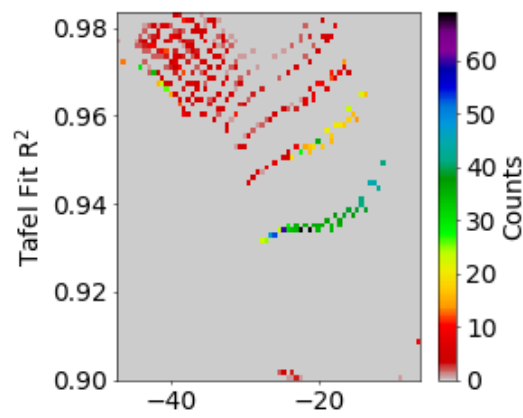

Tafel Slope / $\mathrm{mV}$ decade ${ }^{-1}$

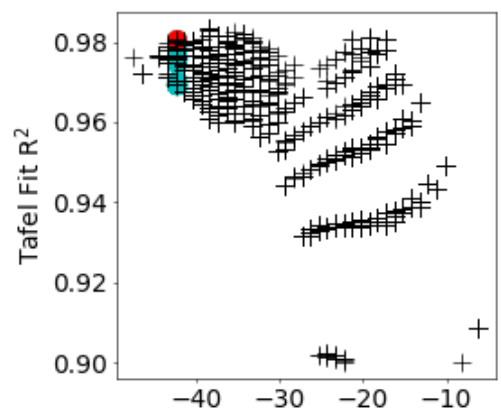

Tafel Slope Bin / mV decade ${ }^{-1}$

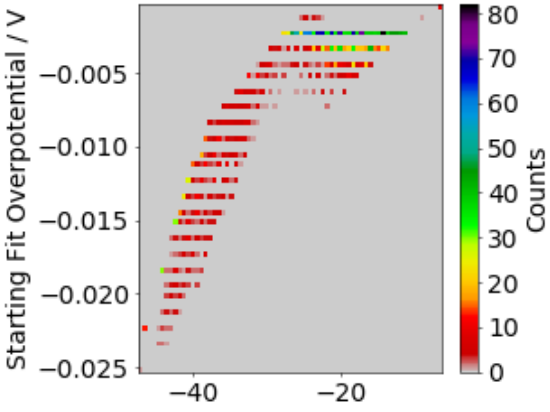

Tafel Slope / $\mathrm{mV}$ decade de $^{-1}$

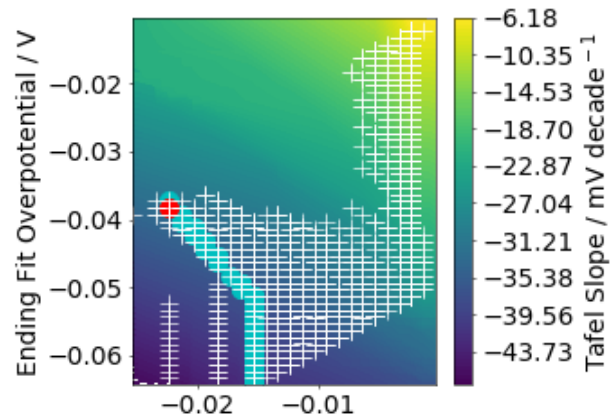

Starting Fit Overpotential / V

Figure S24: HER at 900 rpm; optimal fit $=43 \mathrm{mV}$ decade $^{-1}$
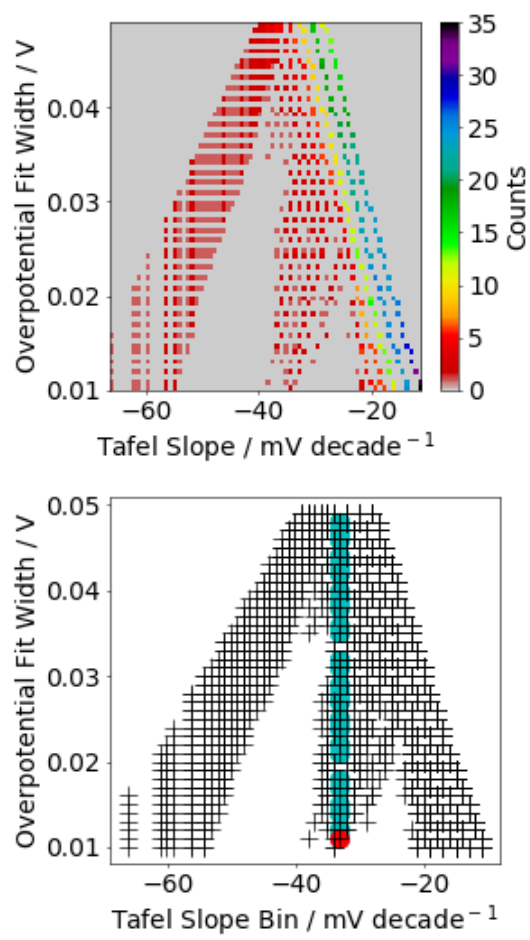
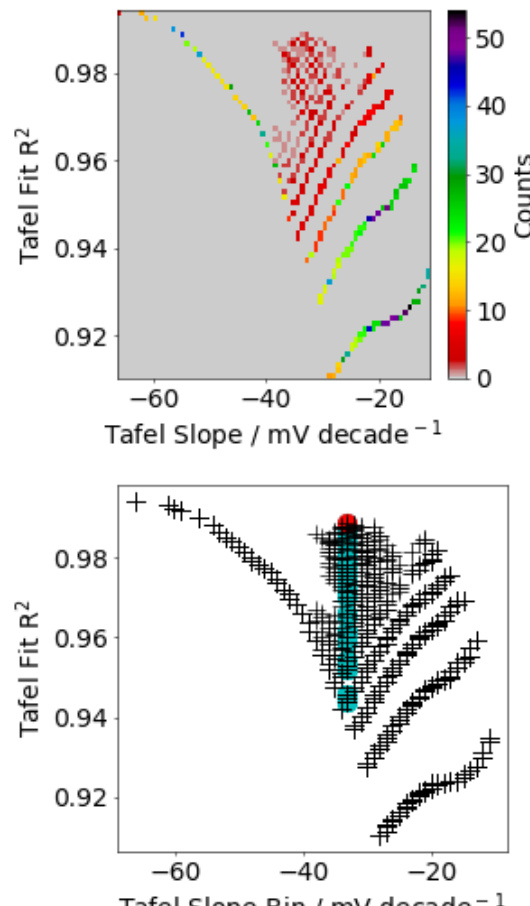

Tafel Slope Bin / mV decade ${ }^{-1}$
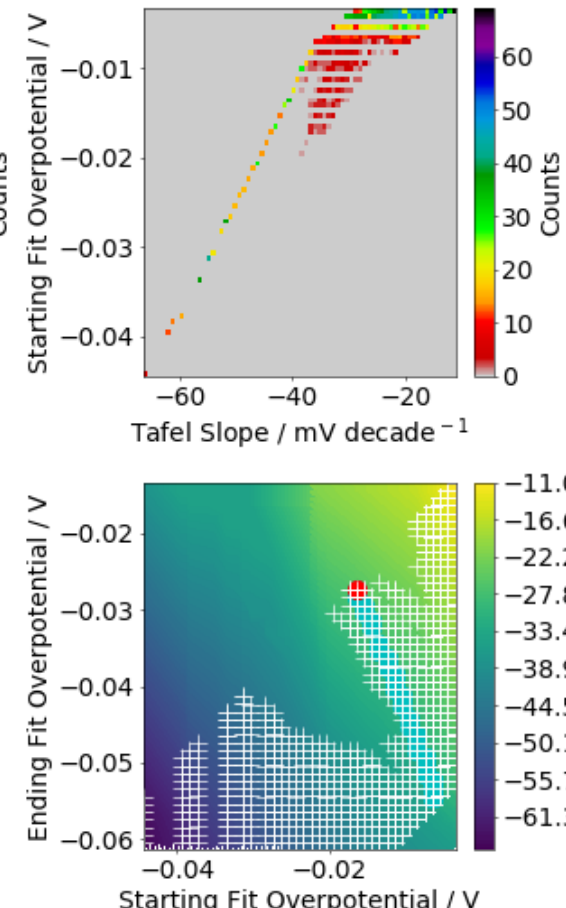

$-11.07$

$-16.65 \ddot{\imath}$

-22.23 은

-27.82 U

$-33.40>$

-38.98 E

-44.56 ฯ

-50.14 은

$-55.72 \frac{\text { \% }}{\alpha}$

$-61.30 \stackrel{\frac{4}{\pi}}{\models}$

Figure S25: HER at $1600 \mathrm{rpm}$; optimal fit $=34 \mathrm{mV}$ decade $^{-1}$ 


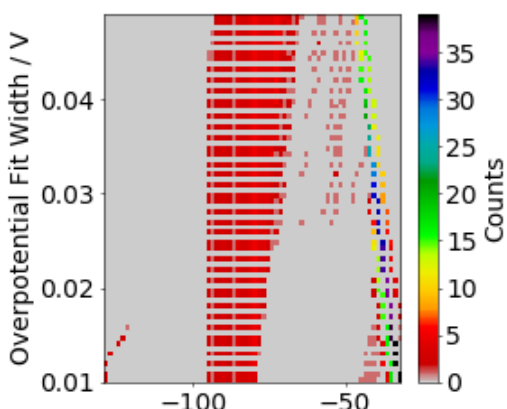

Tafel Slope / $\mathrm{mV}$ decade ${ }^{-1}$

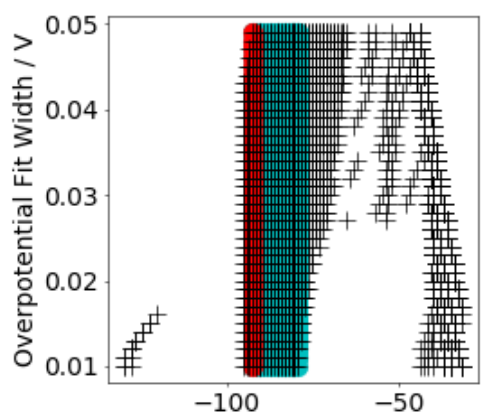

Tafel Slope Bin / mV decade ${ }^{-1}$

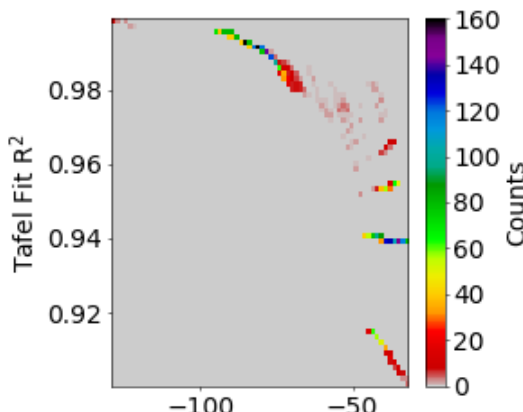

Tafel Slope / $\mathrm{mV}$ decade ${ }^{-1}$

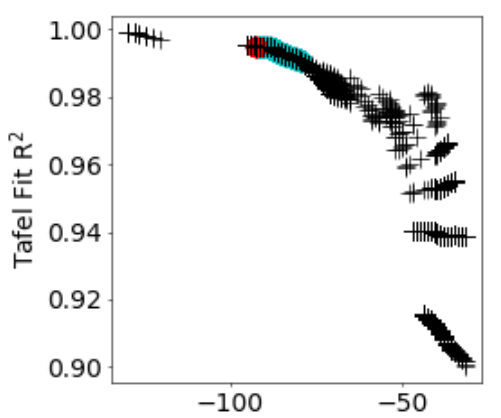

Tafel Slope Bin / mV decade ${ }^{-1}$

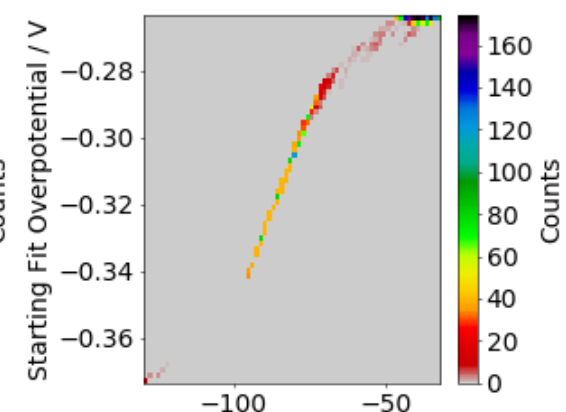

Tafel Slope / $\mathrm{mV}$ decade ${ }^{-1}$

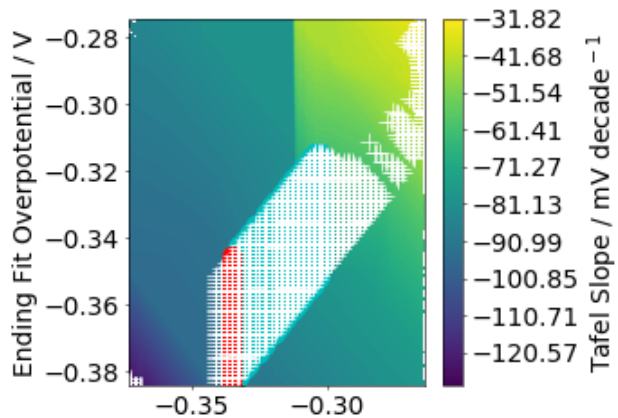

Starting Fit Overpotential / V

Figure S26: ORR at 900 rpm; optimal fit $=93 \mathrm{mV} \mathrm{\text {decade } ^ { - 1 }}$

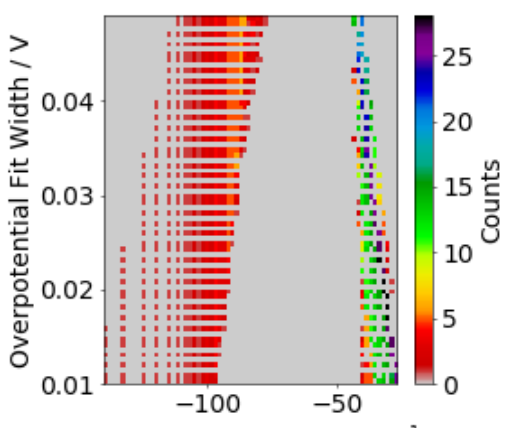

Tafel Slope / $\mathrm{mV}$ decade ${ }^{-1}$

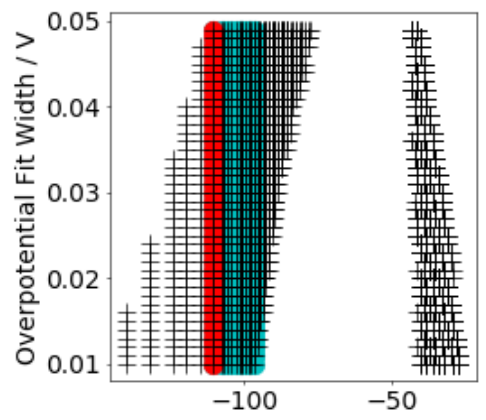

Tafel Slope Bin / mV decade ${ }^{-1}$

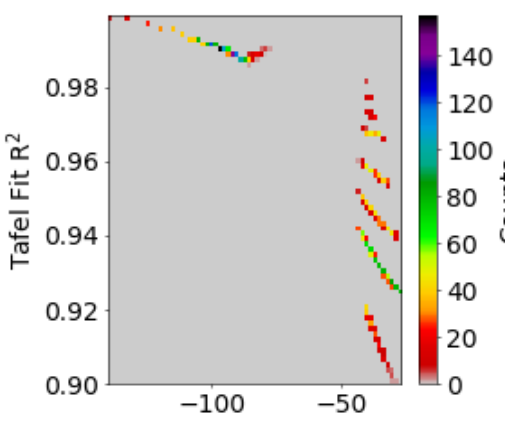

Tafel Slope / $\mathrm{mV}^{\text {decade }}{ }^{-1}$

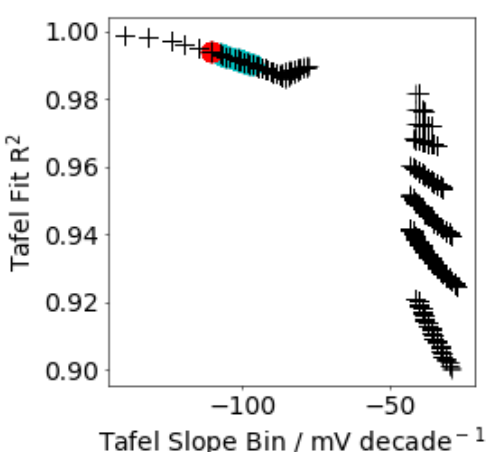

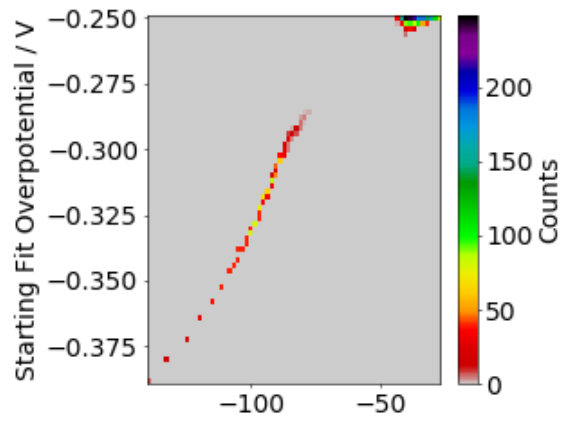

Tafel Slope / $\mathrm{mV}$ decade $^{-1}$

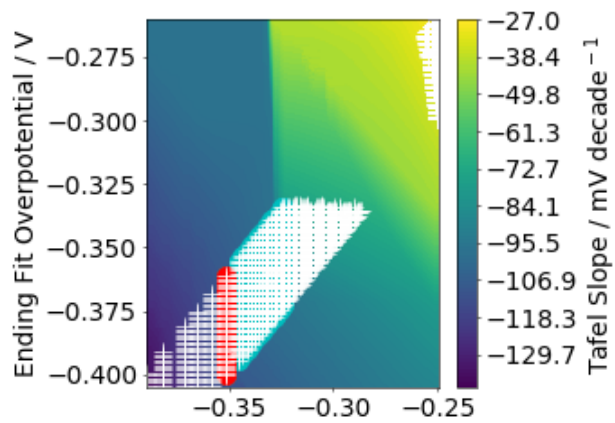

Starting Fit Overpotential / V

Figure S27: ORR at $1600 \mathrm{rpm}$; optimal fit $=111 \mathrm{mV}$ decade $^{-1}$ 


\section{Optimization of the Low-overpotential Tafel Region in ORR}

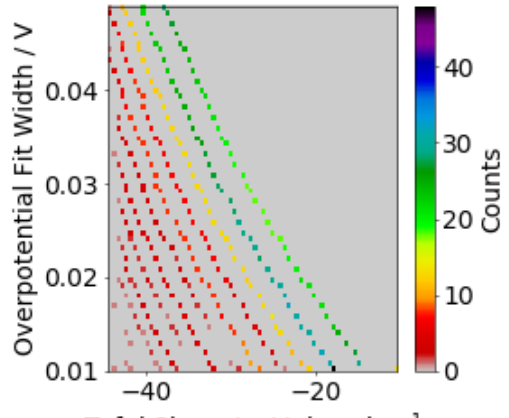

Tafel Slope / $\mathrm{mV}$ decade ${ }^{-1}$

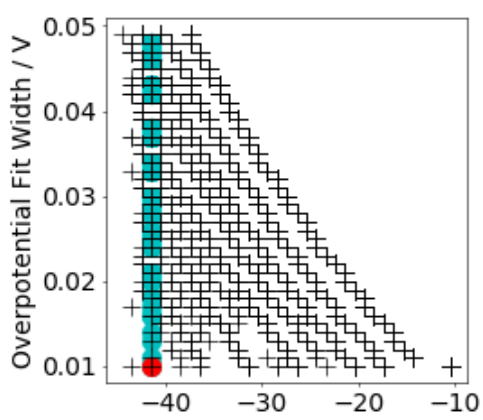

Tafel Slope Bin / $\mathrm{mV}$ decade ${ }^{-1}$

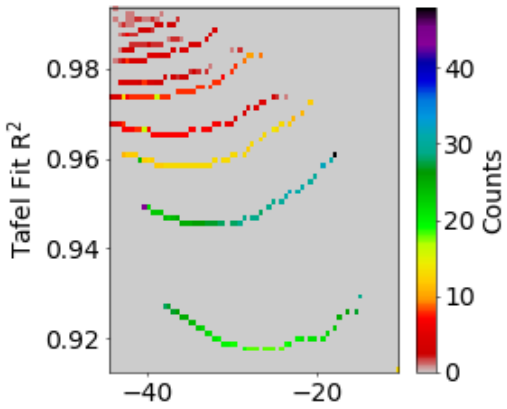

Tafel Slope / $\mathrm{mV}$ decade ${ }^{-1}$

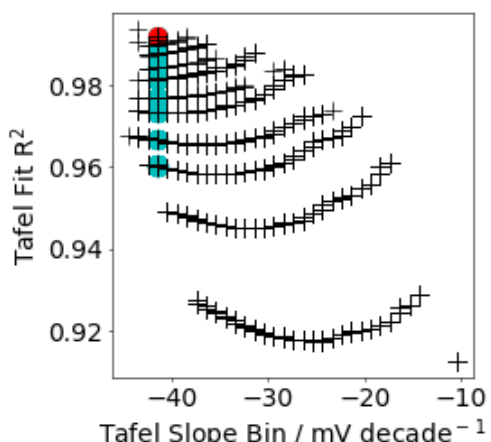

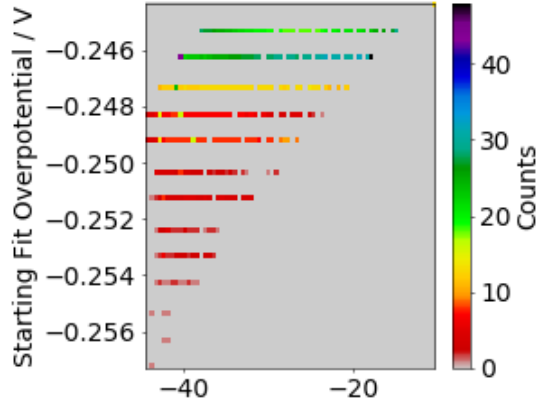

Tafel Slope / $\mathrm{mV}$ decade ${ }^{-1}$

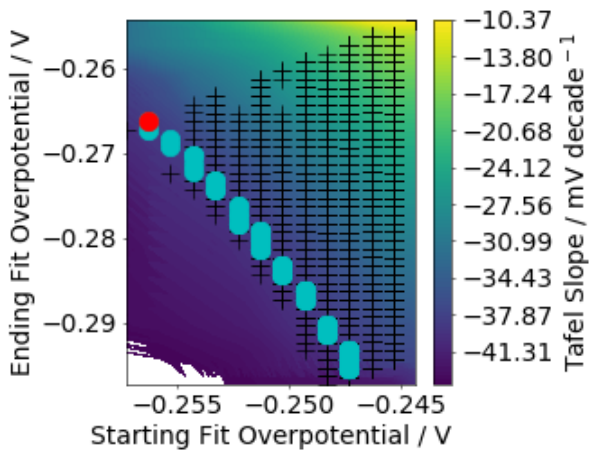

Figure S28: ORR at $2500 \mathrm{rpm}$; binned at $1 \mathrm{mV}$ decade $^{-1}$; optimal fit $=42 \mathrm{mV}$ decade $^{-1}$ 
Table of Binned Data for OER(1600 rpm), HER (2500 rpm), and ORR(2500 rpm)

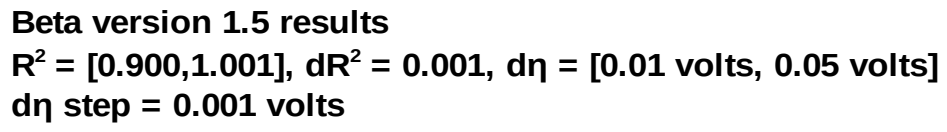

HER (n=1), 2500 rpm

Bin Size (mV/decade)

Tafel Slope (mV/decade)

$\mathrm{I}_{0}(\mathrm{~A})$

2.85E-04

2.85E-04

2.85E-04

2.85E-04

2.85E-04

2.85E-04

2.72E-04

2.85E-04

2.85E-04

2.85E-04

2.84E-04

Average

Std Deviation

ORR (n=1), $2500 \mathrm{rpm}$

Bin Size (mV/decade)

1
2
3
4
5
6
7
8
9
10
Derage

Table S3

\section{Std Deviation}

$\mathrm{I}_{0}(\mathrm{~A})$
$1.87 \mathrm{E}-07$
$1.87 \mathrm{E}-07$
$1.87 \mathrm{E}-07$
$1.87 \mathrm{E}-07$
$1.87 \mathrm{E}-07$
$1.87 \mathrm{E}-07$
$1.87 \mathrm{E}-07$
$2.50 \mathrm{E}-07$
$2.50 \mathrm{E}-07$
$2.50 \mathrm{E}-07$
$2.06 \mathrm{E}-07$
$2.92 \mathrm{E}-08$
(

$-118.93$

$-118.93$

$-118.93$

$-118.93$

$-118.93$

$-118.93$

$-118.93$

$-123.64$

$-123.64$

$-123.64$

120.34

2.16 